\title{
Distributed Generalized Nash Equilibrium Seeking for Energy Sharing Games in Prosumers.
}

\author{
Wang, Zhaojian; Liu, Feng; Ma, Zhiyuan; Chen, Yue; Jia, Mengshuo ; Wei, Wei; Wu, Qiuwei
}

Published in:

IEEE Transactions on Power Systems

Link to article, DOI:

10.1109/TPWRS.2021.3058675

Publication date:

2021

Document Version

Peer reviewed version

Link back to DTU Orbit

Citation (APA):

Wang, Z., Liu, F., Ma, Z., Chen, Y., Jia, M., Wei, W., \& Wu, Q. (2021). Distributed Generalized Nash Equilibrium Seeking for Energy Sharing Games in Prosumers. IEEE Transactions on Power Systems, 36(5), 3973 - 3986. https://doi.org/10.1109/TPWRS.2021.3058675

\section{General rights}

Copyright and moral rights for the publications made accessible in the public portal are retained by the authors and/or other copyright owners and it is a condition of accessing publications that users recognise and abide by the legal requirements associated with these rights.

- Users may download and print one copy of any publication from the public portal for the purpose of private study or research.

- You may not further distribute the material or use it for any profit-making activity or commercial gain

- You may freely distribute the URL identifying the publication in the public portal 


\title{
Distributed Generalized Nash Equilibrium Seeking for Energy Sharing Games in Prosumers
}

\author{
Zhaojian Wang, Feng Liu, Zhiyuan Ma, Yue Chen, Mengshuo Jia, Wei Wei, and Qiuwei Wu
}

\begin{abstract}
With the proliferation of distributed generators and energy storage systems, traditional passive consumers in power systems have been gradually evolving into the so-called "prosumers", i.e., proactive consumers, which can both produce and consume power. To encourage energy exchange among prosumers, energy sharing is increasingly adopted, which is usually formulated as a generalized Nash game (GNG). In this paper, a distributed approach is proposed to seek the Generalized Nash equilibrium (GNE) of the energy sharing game. To this end, we first prove the strong monotonicity of the game. Then, the GNG is converted into an equivalent optimization problem. An algorithm based on Nesterov's methods is thereby devised to solve the equivalent problem and consequently find the GNE in a distributed manner. The convergence of the proposed algorithm is proved rigorously based on the nonexpansive operator theory. The performance of the algorithm is validated by experiments with three prosumers, and the scalability is tested by simulations using 1888 prosumers.
\end{abstract}

Index Terms-Energy sharing, prosumer, distributed algorithm, generalized Nash game, generalized Nash Equilibrium.

\section{NOMENCLATURE}

\section{A. Variables and Functions}

$\begin{array}{ll}p_{i} & \text { Power generation of prosumer } i \\ b_{i} & \text { Purchase willingness of prosumer } i \\ q_{i} & \text { Power shortage/surplus of prosumer } i \\ \mu_{c} & \text { Market clearing price } \\ \lambda_{i}, \kappa_{i} & \text { Lagrangian multipliers } \\ \mu_{i} & \text { Local estimation of } \mu_{c} \text { by prosumer } i \\ z_{i} & \text { A variable to drive the consensus of } \mu_{i} \\ (\cdot)_{i, t} & \text { The variable }(\cdot)_{i} \text { at iteration } t \\ (\widetilde{*})_{i, t} & \text { Temporary variable to estimate }(\cdot)_{i} \text { at iteration } t \\ & \\ h_{i}\left(p_{i}\right) & \text { Cost of prosumer } i \text { to produce } p_{i} \\ f_{i}\left(p_{i}, \boldsymbol{b}\right) & \text { Disutility function of prosumer } i\end{array}$

This work was supported by the National Natural Science Foundation of China ( No. 51677100, U1966601, U1766206 ). (Corresponding author: Feng Liu)

Z. Wang is with the Key Laboratory of System Control, and Information Processing, Ministry of Education of China, Department of Automation, Shanghai Jiao Tong University, Shanghai, 200240, China, and also with the State Key Laboratory of Power System, Department of Electrical Engineering, Tsinghua University, Beijing 100084, China

F. Liu, Y. Chen, M. Jia, and W. Wei are with the State Key Laboratory of Power System and the Department of Electrical Engineering, Tsinghua University, Beijing, 100084, China (e-mail: lfeng@tsinghua.edu.cn).

Z. Ma is with the Global Energy Interconnection Development and Cooperation Organization, Beijing, 100031, China.

Q. Wu is with the Center for Electric Power and Energy, Department of Electrical Engineering, Technical University of Denmark, Kgs. Lyngby, DK 2800, Denmark. $g_{i}(\boldsymbol{b}) \quad$ Cost of prosumer $i$ to buy/sell power from/to others

$F(p, b) \quad$ Pseudo-gradient of $f_{i}\left(p_{i}, \boldsymbol{b}\right), \forall i \in \mathcal{N}$

$G(\boldsymbol{b}) \quad$ Pseudo-gradient of $g_{i}(\boldsymbol{b}), \forall i \in \mathcal{N}$

$\tilde{h}(\boldsymbol{p}) \quad$ Objective function of the equivalent optimization problem

$\tilde{h}_{i}\left(p_{i}\right) \quad$ Objective function of the equivalent optimization problem related to prosumer $i$

\section{B. Parameters and Matrices}

$D_{i}$
$a_{i}$
$a_{\Sigma}$
$\underline{p}_{i}, \bar{p}_{i}$
$\gamma_{i}, \sigma_{z}, \sigma_{\mu}, \eta$
$(\cdot)^{*}$
$\alpha_{i}$
$\beta_{i}$
$\delta_{i}$
$\tilde{h}_{0}$

Load demand of prosumer $i$

Price elasticity of prosumer $i$

The sum of $a_{i}, \forall i$

Lower/upper bound of $p_{i}$

Positive stepsizes

The value of $(\cdot)$ at the equilibrium

Constant determined by $\alpha_{i}=\left(a_{i}-a_{\Sigma}\right) / a_{\Sigma}^{2}$

Constant determined by $\beta_{i}=\left(2 a_{i}-a_{\Sigma}\right) / a_{\Sigma}^{2}$

Constant determined by $\delta_{i}=a_{i} / a_{\Sigma}^{2}$

Positive constant determined by the strong convexity of function $\tilde{h}_{i}\left(p_{i}\right)$

$L \quad$ Laplacian matrix of the communication graph

$I_{n} \quad$ Identity matrix with dimension $n$

$\Gamma \quad$ Diagonal matrix, $\Gamma=\operatorname{diag}\left(\gamma_{i}\right), \forall i$

$E_{n} \quad n$-dimension Matrix with all of elements as 1

\section{Sets and Operators}

$\mathcal{N} \quad$ Set of prosumers

$\mathcal{E} \quad$ Set of comunication edges of prosumers

$N_{i} \quad$ Set of neighbors of prosumers $i$ over the communication graph

$\Omega_{i} \quad$ Set of generation limits of prosumer $i$

$\Omega \quad$ Cartesian product of $\Omega_{i}, i \in \mathcal{N}$

$X_{i}^{e} \quad$ Set of the local power balance constraint of prosumer $i$

$X^{e} \quad$ Cartesian product of $X_{i}^{e}, i \in \mathcal{N}$

$X_{i} \quad$ Intersection of $\Omega_{i}$ and $X_{i}^{e}$

$X \quad$ Intersection of $\Omega$ and $X^{e}$

$\mathcal{P}_{\Omega}(\boldsymbol{x}) \quad$ Projection of $x$ onto the set $\Omega$

Id Identity operator

$N_{\Omega}(\boldsymbol{x}) \quad$ Normal cone of $\Omega$ at the point $\boldsymbol{x}$

$\mathcal{U}$ Operator derived from the algorithm, defined in (27)

$\operatorname{diag}(x)$ Diagonal matrix with $x$ as the element 


\section{Abbreviations}

$\begin{array}{ll}\text { DG } & \text { Distributed generation } \\ \text { GNG } & \text { Generalized Nash game } \\ \text { GNE } & \text { Generalized Nash equilibrium } \\ \text { ADMM } & \text { Alternating direction method of multipliers } \\ \text { SGNE } & \text { Seeking the Generalized Nash Equilibrium }\end{array}$

\section{INTRODUCTION}

It has been recognized that our power system is undergoing a fundamental transition due to: 1) The proliferation of distributed generations (DGs), such as wind turbines, photovoltaics, energy storage systems and electric vehicles [1]-[5]; 2) The advancement of communication and control technologies in the consumer-level via smart appliances and energy management systems [6]-[8]. Together, these changes allow traditional passive consumers to evolve into the so-called "prosumers", i.e., proactive consumers, which can actively regulate their generation and consumption [9]-[12]. Conventionally, a hierarchical structure is utilized in the power system energy management, usually in a centralized way. However, the centralized manner may face great challenges raised by the ever-increasing number of prosumers and fast variation of renewable generations. This essentially advocates a distributed paradigm [13]-[16]. Particularly, energy sharing turns to be a promising form of the market that encourages energy trading among prosumers.

For energy sharing, the existing literature can be roughly cast into two categories. 1) Price guided energy sharing. There are usually two mechanisms for setting the price. The first one is the energy sharing with set price, where the sharing price is constant or determined by the platform [17]-[26]. Prosumers solve decision making problems to adjust power generation or consumption. The second type is the energy sharing with clearing price, where sellers and buyers bid for some amount of products [27]-[31]. Then, the platform solves an optimization problem to maximize social welfare or self-revenue with these bids and clears the market. 2) Utility reallocation based energy sharing. In this kind of sharing, the key point is to design schemes for the allocation of profit/cost [32]-[35]. Some renowned mechanisms include Vickrey-Clarke-Groves and coalitional game approaches.

The energy sharing mechanism in this paper is derived from that of our previous work [30], which has two advantages compared with existing literature. 1) Each prosumer is symmetric, whose role is not predetermined as a buyer or a seller. In the existing literature, the prosumer is assigned as a buyer or a seller in advance by the prediction of its power generation and consumption [17]-[29]. 2) The participation of prosumers is self-guided. The market operator only broadcasts the sum of price elasticity instead of computing prices. In many existing works, the platform declares the price and prosumers act only as price-takers [17]-[26]. The proposed model provides sufficient autonomy for prosumers, which can encourage their participation in the energy sharing. Because prosumers typically belong to different owners, the inherent competition may lead to strategic behaviors in energy sharing. In this situation, each prosumer intends to optimize its profit while maintaining the power balance over the whole system. This leads to a generalized Nash game (GNG) with global constraints. In this regard, it is desirable to investigate distributed approaches to seeking the generalized Nash equilibrium (GNE) of the GNG.

There are many papers investigating distributed methods of GNE seeking, which can be roughly divided into two categories in terms of methods used: gradient-based algorithms [36]-[41], and proximal-point algorithms [42]-[45]. In the first type, the pseudo-gradient of each player's disutility function is utilized to seek the GNE, including continuous-time algorithms [36], [37] and discrete algorithms [38]-[41]. In [36], a distributed continuous-time projection-based algorithm is proposed to seek the GNE of aggregative games with linear coupled constraints. This is extended to the case where players have nonsmooth payoff functions in [37]. In [38], two distributed primal-dual algorithms are proposed for computing a GNE in noncooperative games with shared affine constraints. The operator splitting method is utilized to prove the convergence of the algorithms. It is further improved in [39] to consider communication time delays and partial-decision information. In [40], three stochastic gradient strategies are developed to seek GNE where agents are subject to randomness in the environment of unknown statistical distribution. In [41], the relations between Nash and Wardrop equilibria of the aggregative game are investigated and two algorithms are proposed to seek the equilibrium.

In the proximal-point algorithms, the Alternating Direction Method of Multipliers (ADMM) is widely used. An inexactADMM algorithm is proposed in [42]. This method is improved in [45], where each player only has partial information of their opponents and the communication graph is not necessarily the same as the cost dependency graph of each player. In [43], two double-layer preconditioned proximalpoint algorithms are proposed to seek GNE with both coupled equality and inequality constraints, respectively. For the GNG with a special structure, i.e., the coupling in the cost functions of the agents is linear, a distributed proximal-point algorithm is developed in [44] for GNG with maximally monotone pseudogradient.

The aforementioned works have made great progress in the context of distributed GNE seeking. In most of the works, if the objective function of one player is associated with the decisions of all players, each player is required to communicate with all of the other players, i.e., full information is needed. Then, the communication network is very dense. To address this problem, a local estimation of the overall decision profile for each player is added in [39], [46]. However, this will increase one order in the algorithm, making it more complicated.

In this paper, we offer a different perspective for seeking the GNE in the GNG when full information is included in the objective functions. The energy sharing game is transformed into an equivalent optimization problem. Instead of dealing with the GNG directly, we alternatively solve the equivalent problem. Both experiments and simulations are utilized to validate our method. The main contributions are as follows.

- We first prove that the energy sharing game is strongly monotone, i.e., the pseudo-gradient of the objective func- 
tion is strongly monotone. The traditional method solving such strongly monotone games requires that each player has the unified label of all $n$ players and introduces an extra variable with $n-1$ dimension [46], [47]. This is almost impossible to realize in a distributed way, which motivates us to develop a totally different way to solve the game.

- A systematic way based on the variational inequality is proposed to transform the energy sharing game into an equivalent centralized optimization problem, which could be solved in a fully distributed way with neighboring communication. Moreover, the existence and uniqueness of the GNE of the energy sharing game are proved.

- A distributed algorithm is devised to solve the equivalent counterpart instead of solving the original game problem directly. In this way, each prosumer only needs to know its neighbors and update a 6-dimension variable, which is much simpler and more practical. By constructing a firmly nonexpansive operator, we prove that the proposed distributed algorithm converges to the GNE of the original energy sharing game.

The rest of this paper is organized as follows. In Section II, we briefly introduce some necessary preliminaries. Section III formulates the energy sharing model. In Section IV, the existence and uniqueness of GNE are analyzed. A distributed GNE seeking algorithm is proposed in Section V. The convergence of the algorithm is proved in Section VI. The effectiveness of the algorithm is verified in Section VII by experiments and simulation studies. Section VIII concludes the paper.

\section{PRELIMINARIES}

In this paper, use $\mathbb{R}^{n}$ to denote the $n$-dimension Euclidean space. For a matrix $A,\left[A_{i j}\right]$ is the entry in the $i$-th row and $j$-th column of $A$. For vectors $\boldsymbol{x}, \boldsymbol{y} \in \mathbb{R}^{n}, \boldsymbol{x}^{\mathrm{T}} \boldsymbol{y}=\langle\boldsymbol{x}, \boldsymbol{y}\rangle$ denotes the inner product of $x, y \cdot\|\boldsymbol{x}\|_{2}=\sqrt{\boldsymbol{x}^{\mathrm{T}} \boldsymbol{x}}$ denotes the Euclidean norm of $\boldsymbol{x}$. Denote the inner product under a positive definite matrix $Q$ by $\langle\boldsymbol{x}, \boldsymbol{y}\rangle_{Q}=\langle Q \boldsymbol{x}, \boldsymbol{y}\rangle$. Similarly, the norm induced by $Q$ is $\|\boldsymbol{x}\|_{Q}=\sqrt{\langle Q \boldsymbol{x}, \boldsymbol{x}\rangle}$. The following relationship holds for a $Q$-induced norm.

$$
\|\boldsymbol{a}-\boldsymbol{c}\|_{Q}^{2}-\|\boldsymbol{b}-\boldsymbol{c}\|_{Q}^{2}=2\langle\boldsymbol{a}-\boldsymbol{b}, \boldsymbol{a}-\boldsymbol{c}\rangle_{Q}-\|\boldsymbol{a}-\boldsymbol{b}\|_{Q}^{2}
$$

which can be obtained by the equation $\|\boldsymbol{a}+\boldsymbol{b}\|_{Q}^{2}=\|\boldsymbol{a}\|_{Q}^{2}+$ $2\langle\boldsymbol{a}, \boldsymbol{b}\rangle_{Q}+\|\boldsymbol{b}\|_{Q}^{2}$.

The identity matrix with dimension $n$ is denoted by $I_{n}$. Use $\prod_{i=1}^{n} \Omega_{i}$ to denote the Cartesian product of the sets $\Omega_{i}, i=$ $1, \cdots, n$. Define the projection of $x$ onto a set $\Omega$ as

$$
\mathcal{P}_{\Omega}(\boldsymbol{x})=\arg \min _{\boldsymbol{y} \in \Omega}\|\boldsymbol{x}-\boldsymbol{y}\|_{2}
$$

Use Id to denote the identity operator, i.e., $\operatorname{Id}(\boldsymbol{x})=\boldsymbol{x}, \forall \boldsymbol{x}$. Define $N_{\Omega}(\boldsymbol{x})=\{\boldsymbol{v} \mid\langle\boldsymbol{v}, \boldsymbol{y}-\boldsymbol{x}\rangle \leq 0, \forall \boldsymbol{y} \in \Omega\}$. We have $\mathcal{P}_{\Omega}(\boldsymbol{x})=\left(\operatorname{Id}+N_{\Omega}\right)^{-1}(\boldsymbol{x})$ [48, Chapter 23.1].

For a single-valued operator $\mathcal{T}: \Omega \subset \mathbb{R}^{n} \rightarrow \mathbb{R}^{n}$, a point $\boldsymbol{x} \in \Omega$ is a fixed point of $\mathcal{T}$ if $\mathcal{T}(\boldsymbol{x}) \equiv \boldsymbol{x}$. The set of fixed points of $\mathcal{T}$ is denoted by $\operatorname{Fix}(\mathcal{T}) . \mathcal{T}$ is nonexpansive if $\|\mathcal{T}(\boldsymbol{x})-\mathcal{T}(\boldsymbol{y})\| \leq\|\boldsymbol{x}-\boldsymbol{y}\|, \forall \boldsymbol{x}, \boldsymbol{y} \in \Omega$. For $\alpha \in(0,1), \mathcal{T}$ is called $\alpha$-averaged if there exists a nonexpansive operator $\mathcal{R}$ such that $\mathcal{T}=(1-\alpha) \operatorname{Id}+\alpha \mathcal{R}$. Use $\mathcal{A}(\alpha)$ to denote the class of $\alpha$-averaged operators. If
$\mathcal{T} \in \mathcal{A}\left(\frac{1}{2}\right), \mathcal{T}$ is called firmly nonexpansive. The graph of $\mathcal{T}$ is gra $\mathcal{T}=\left\{(\boldsymbol{x}, \boldsymbol{u}) \in \mathbf{R}^{n} \times \mathbf{R}^{n} \mid \boldsymbol{u} \in \mathcal{T}(\boldsymbol{x})\right\} . \mathcal{T}$ is monotone if $\forall(\boldsymbol{x}, \boldsymbol{u}), \forall(\boldsymbol{y}, \boldsymbol{v}) \in \operatorname{gra} \mathcal{T},\langle\boldsymbol{x}-\boldsymbol{y}, \boldsymbol{u}-\boldsymbol{v}\rangle \geq 0 . \mathcal{T}$ is maximally monotone if gra $\mathcal{T}$ is not strictly contained in the graph of any other monotone operator.

\section{ENERGY SHARING GAME}

In this section, the model of prosumers is introduced. Then, we formulate the energy sharing game.

\section{A. Model of prosumers}

In this paper, we consider the energy sharing problem among a set of prosumers, denoted by $\mathcal{N}=\{1,2, \ldots n\}$. The communication edge is denoted by $\mathcal{E} \subseteq \mathcal{N} \times \mathcal{N}$. For a prosumer $i$, the set of its neighbors is denoted by $N_{i}$. If $j \in N_{i}$, prosumers $i$ and $j$ can communicate directly. The Laplacian matrix of the communication graph is denoted by $L$ and we have $\mathbf{1}^{\mathrm{T}} L=0$, where $\mathbf{1}$ is a vector with all of the components as 1 .

The scenario is that each prosumer has load demand, denoted by $D_{i}$. To maintain the power balance, it can produce power, denoted by $p_{i}$, or buy power from (sell power to) the grid, denoted by $q_{i}$. In the sharing market, the demand function of each prosumer can be expressed by

$$
q_{i}=a_{i} \mu_{c}+b_{i}
$$

where $\mu_{c}$ is the market clearing price, and $b_{i}$ is the willingness to buy. $a_{i}<0$ implies the price sensitivity. The market clears when the net quantity $\sum_{i \in \mathcal{N}} q_{i}=0$ and the sharing price is ${ }^{1}$

$$
\mu_{c}=-\frac{\mathbf{1}^{\mathrm{T}} \boldsymbol{b}}{a_{\Sigma}}
$$

where $a_{\Sigma}=\sum_{i \in \mathcal{N}} a_{i}$ is the sum of $a_{i}$. In the rest of the paper, $\boldsymbol{b}_{-i}:=\left(b_{1}, b_{2}, \cdots, b_{i-1}, b_{i+1}, \cdots, b_{n}\right)^{\mathrm{T}}$.

\section{B. Energy sharing game}

In the energy sharing game, each prosumer intends to minimize its cost while maintaining the global power balance. The optimization problem of each prosumer is

$$
\begin{aligned}
\min _{p_{i}, b_{i}} & f_{i}\left(p_{i}, \boldsymbol{b}\right)=h_{i}\left(p_{i}\right)+\left(a_{i} \mu_{c}(\boldsymbol{b})+b_{i}\right) \mu_{c}(\boldsymbol{b}) \\
\text { s.t. } & p_{i}+a_{i} \mu_{c}(\boldsymbol{b})+b_{i}=D_{i} \\
& \sum_{i \in \mathcal{N}}\left(a_{i} \mu_{c}(\boldsymbol{b})+b_{i}\right)=0 \\
& \underline{p}_{i} \leq p_{i} \leq \bar{p}_{i}
\end{aligned}
$$

The disutility function consists of two parts, where $h_{i}\left(p_{i}\right)$ is the cost of prosumer $i$ to produce $p_{i}$ and $\left(a_{i} \mu_{c}(\boldsymbol{b})+b_{i}\right) \mu_{c}(\boldsymbol{b})=$ $q_{i} \mu_{c}(\boldsymbol{b})$ is the cost of buying power $q_{i}$.

The Lagrangian of (5) is

$$
\begin{aligned}
& \mathcal{L}\left(p_{i}, \boldsymbol{b}, \lambda_{i}, \kappa_{i}\right)=f_{i}\left(p_{i}, \boldsymbol{b}\right)+\lambda_{i}\left(p_{i}+a_{i} \mu_{c}(\boldsymbol{b})+b_{i}-D_{i}\right) \\
& \quad \underline{p}_{i} \leq p_{i} \leq \bar{p}_{i}, \boldsymbol{b}_{i} \\
& \quad+\kappa_{i}\left(\sum_{i \in \mathcal{N}}\left(a_{i} \mu_{c}(\boldsymbol{b})+b_{i}\right)\right)
\end{aligned}
$$

\footnotetext{
${ }^{1}$ Given a collection of $z_{i}$ for $i$ in a certain set $Z, \boldsymbol{z}$ denotes the column vector $\boldsymbol{z}:=\operatorname{col}\left(z_{i}, i \in Z\right)$ with a proper dimension with $z_{i}$ as its components.
} 
where $\lambda_{i}, \kappa_{i}$ are Lagrangian multipliers.

Regarding the energy sharing game (5), we have following assumptions.

Assumption 1. The functions $h_{i}\left(p_{i}\right), \forall i \in \mathcal{N}$ are convex and differentiable.

Assumption 2. For a given $\boldsymbol{b}_{-i}$, the problem (5) is feasible.

Assumption 3. The generation satisfies $\mathbf{1}^{\mathrm{T}} p<\mathbf{1}^{\mathrm{T}} \boldsymbol{D}<\mathbf{1}^{\mathrm{T}} \overline{\boldsymbol{p}}$.

For Assumption 1, the power generation cost $h_{i}\left(p_{i}\right)$ is usually quadratic, which is convex and differentiable. For Assumption 2, because the constraints of problem (5) are all affine equalities and inequalities, the Slater's condition of (5) reduces to its feasibility [49, Page $226 \&$ 227]. Thus, Assumption 2 is a common assumption, otherwise, the problem is infeasible. Assumption 3 implies that energy production can strictly satisfy the load demand. As long as the total regulation capacity of all prosumers is strictly larger than the total load demand, Assumption 3 holds. Assumption 3 is stricter than Assumption 2, which is used to determine the uniqueness of the GNE.

In summary, the energy sharing game among all prosumers is composed of the following elements:

- Player: all prosumers, denoted by $\mathcal{N}=\{1,2, \ldots n\}$;

- Strategy: generation power $p_{i}$ and power interaction $b_{i}$;

- Payoff: the disutility function $f_{i}\left(p_{i}, \boldsymbol{b}\right), \forall i \in \mathcal{N}$.

The GNE $\left(\boldsymbol{p}^{*}, \boldsymbol{b}^{*}\right)$ of the game in (5) is defined as:

$\left(p_{i}^{*}, b_{i}^{*}\right) \in \arg \min f_{i}\left(p_{i}, b_{i}, \boldsymbol{b}_{-i}^{*}\right)$, s.t. (5b), (5b), (5c), $\forall i \in \mathcal{N}$

Remark 1. The energy sharing game model (5) basically comes from [30], and is modified by additionally considering local capacity constraints (5d). Mathematically, the energy sharing game (5) is indeed a GNG by noting that the global constraint $(5 \mathrm{c})$ couples the strategies of players. As proved in [30], if the constraint (5d) is not taken into account, the energy sharing game can be equivalently converted into a standard Nash game instead of a GNG without any coupling constraints. However, when (5d) is considered, the coupling constraints cannot be eliminated. This is the key difference between this paper and [30] in the modeling.

\section{Key Properties of Energy Sharing Game}

In this section, we analyze the property of the Nash equilibrium and transform the game into an equivalent optimization problem.

\section{A. Monotonic analysis}

Replacing $\mu_{c}$ in (5) using (4), then the sharing game problem (5) can be rewritten as

$$
\begin{aligned}
\min _{p_{i}, \boldsymbol{b}_{i}} & f_{i}\left(p_{i}, \boldsymbol{b}\right)=h_{i}\left(p_{i}\right)+g_{i}\left(b_{i}, \boldsymbol{b}_{-i}\right) \\
\text { s.t. } & p_{i}+\frac{a_{\Sigma}-a_{i}}{a_{\Sigma}} b_{i}-\frac{a_{i}}{a_{\Sigma}} \sum_{j \neq i} b_{j}=D_{i} \\
& \underline{p}_{i} \leq p_{i} \leq \bar{p}_{i}
\end{aligned}
$$

where

$$
g_{i}\left(b_{i}, \boldsymbol{b}_{-i}\right)=\left(a_{i} \mu_{c}(\boldsymbol{b})+b_{i}\right) \mu_{c}(\boldsymbol{b})
$$

$$
\begin{aligned}
= & -\left(\frac{a_{\Sigma}-a_{i}}{a_{\Sigma}} b_{i}-\frac{a_{i}}{a_{\Sigma}} \sum_{j \neq i} b_{j}\right) \frac{b_{i}+\sum_{j \neq i} b_{j}}{a_{\Sigma}} \\
= & \frac{a_{i}-a_{\Sigma}}{a_{\Sigma}^{2}} b_{i}^{2}+\left(\frac{2 a_{i}-a_{\Sigma}}{a_{\Sigma}^{2}}\right) b_{i} \sum_{j \neq i} b_{j} \\
& +\frac{a_{i}}{a_{\Sigma}^{2}}\left(\sum_{j \neq i} b_{j}\right)^{2} \\
= & \alpha_{i} b_{i}^{2}+\beta_{i} b_{i} \sum_{j \neq i} b_{j}+\delta_{i}\left(\sum_{j \neq i} b_{j}\right)^{2}
\end{aligned}
$$

with

$$
\alpha_{i}=\frac{a_{i}-a_{\Sigma}}{a_{\Sigma}^{2}}, \beta_{i}=\frac{2 a_{i}-a_{\Sigma}}{a_{\Sigma}^{2}}, \delta_{i}=\frac{a_{i}}{a_{\Sigma}^{2}}
$$

Because $a_{i}<0$, we have $\alpha_{i}>0, \beta_{i}>0, \delta_{i}<0$. Then, $g_{i}\left(b_{i}, \boldsymbol{b}_{-i}\right)$ is strictly convex with respect to $b_{i}$ given any $\boldsymbol{b}_{-i}$.

The pseudo-gradient of $f_{i}\left(p_{i}, \boldsymbol{b}\right), \forall i \in \mathcal{N}$, denoted by $F(p, b)$, is defined as

$$
\begin{aligned}
F(\boldsymbol{p}, \boldsymbol{b})= & \operatorname{col}\left(\nabla_{p_{1}, b_{1}} f_{1}\left(p_{1}, \boldsymbol{b}\right), \cdots, \nabla_{p_{N}, b_{N}} f_{N}\left(p_{N}, \boldsymbol{b}\right)\right) \\
= & \operatorname{col}\left(\nabla_{p_{1}} h_{1}\left(p_{1}\right), \cdots, \nabla_{p_{N}} h_{N}\left(p_{N}\right)\right) \\
& +\operatorname{col}\left(\nabla_{b_{1}} g_{1}(\boldsymbol{b}), \cdots, \nabla_{b_{N}} g_{N}(\boldsymbol{b})\right)
\end{aligned}
$$

Regarding the pseudo-gradient $F(\boldsymbol{p}, \boldsymbol{b})$, we have the following results.

Theorem 1. Suppose Assumption 1 and 2 hold. The pseudogradient $F(\boldsymbol{p}, \boldsymbol{b})$ is strongly monotone.

The proof of Theorem 1 is introduced in Appendix A.

Because the pseudo-gradient $F(\boldsymbol{p}, \boldsymbol{b})$ is strongly monotone, game (7) could be solved directly by many algorithms in the existing literature. However, the objective function $g_{i}\left(b_{i}, \boldsymbol{b}_{-i}\right)$ is associated with all the players. When solving (7) directly, each player is required to have access to the decisions of all opponents, i.e., full information is needed. This is very difficult to implement in practice. Recently, some papers propose a consensus-based algorithm [46], [47], where each player has an extra proportional term of the consensual errors between his decisions and estimates of his neighbors. Then, only communications with neighbors are needed. However, there are three obstacles to use this method. First, each player needs to know the total amount of players in order to construct the consensus variable. Second, each player needs to have the unified label of other players in order to implement the consensus operation. Third, each player should have estimates of all of the other opponents, which introduces an extra variable with $n-1$ dimension. To overcome the first two obstacles, a control center is needed to label all of the players. In addition, when some prosumers join or leave, all of the other prosumers need to change the dimension of their state variables. All of these will increase the computation and communication burden, which restricts the implementation of the method. In the rest of this work, we give a different perspective by solving an equivalent optimization problem of (7).

\section{B. Equivalent Problem and Uniqueness of GNE}

Define sets

$$
\Omega_{i}:=\left\{p_{i} \mid \underline{p}_{i} \leq p_{i} \leq \bar{p}_{i}\right\}, \Omega=\prod_{i=1}^{n} \Omega_{i}
$$


and

$$
\begin{gathered}
X_{i}^{e}:=\left\{\left(p_{i}, b_{i}\right) \mid p_{i}+\frac{a_{\Sigma}-a_{i}}{a_{\Sigma}} b_{i}-\frac{a_{i}}{a_{\Sigma}} \sum_{j \neq i} b_{j}=D_{i}\right\} \\
X^{e}=\prod_{i=1}^{n} X_{i}^{e}, X_{i}=\Omega_{i} \cap X_{i}^{e}, X=\Omega \cap X^{e}
\end{gathered}
$$

For any given $\boldsymbol{b}_{-i}$, the feasible set of prosumer $i, X_{i}$, is closed and convex. Then, we have the following result.

Lemma 2. If assumptions 1 and 2 hold, a point $(\boldsymbol{p}, \boldsymbol{b})$ is an equilibrium if and only if it is a solution of the variational inequality $V I(X, F(\boldsymbol{p}, \boldsymbol{b}))^{2}$.

Proof. For any given $\boldsymbol{b}_{-i}$, the disutility function (7a) is continuous, differentiable and convex with respect to $\left(p_{i}, b_{i}\right)$. Moreover, the feasible set $X_{i}$ is closed and convex. Thus, we have this assertion by [50, Theorem 3.3].

For the existence and uniqueness of the GNE, we have the following results.

Theorem 3. If Assumption 1 and Assumption 2 hold, for the generalized Nash game (7), we have

1) the generalized Nash equilibrium exists;

2) if the Assumption 3 also holds, the generalized Nash equilibrium is unique.

The detailed proof of Theorem 3 is given in Appendix B. From the proof of Theorem 3, an equivalent optimization problem is obtained, which is

$$
\begin{array}{ll}
\min _{\boldsymbol{p}} & \tilde{h}(\boldsymbol{p})=\sum_{i \in \mathcal{N}}\left(h_{i}\left(p_{i}\right)+\frac{p_{i}^{2}}{2\left(a_{i}-a_{\Sigma}\right)}-\frac{D_{i}}{a_{i}-a_{\Sigma}} p_{i}\right) \\
\text { s.t. } \quad \sum_{i \in \mathcal{N}} p_{i}=\sum_{i \in \mathcal{N}} D_{i}, \quad \mu_{c} \\
\quad \underline{p}_{i} \leq p_{i} \leq \bar{p}_{i}
\end{array}
$$

where $\mu_{c}$ is the Lagrangian multiplier of constraint (14b). The "equivalent" here means that we can first solve (14) to get $p^{*}, \mu_{c}^{*}$, and then get $b^{*}$ with $b_{i}^{*}=D_{i}-p_{i}^{*}-a_{i} \mu_{c}^{*}$. Finally, the GNE is obtained.

Remark 2. The game (7) is difficult to solve by existing methods due to the full information in the objective function. Now, we can alternatively consider the equivalent counterpart (14), which could be solved in a distributed way as long as $a_{\Sigma}$ is known. In this regard, the market coordinator (or a thirdparty platform) is required to broadcast the sum of $a_{i}$ of all prosumers. That is needed merely when new prosumers join or existing ones quit, which can be known by the market coordinator or the third-party platform. After getting $p_{i}^{*}, b_{i}^{*}$ can be obtained from $b_{i}^{*}=D_{i}-p_{i}^{*}-a_{i} \mu_{c}^{*}$. Then, the energy sharing game problem (7) can be solved.

The method using KKT conditions as the bridge to connect the game model and the optimization problem is also utilized in [51]. Although there are some similarities, our paper has great improvements. In [51], the Cournot game is utilized, where the producer is known in advance. The KKT condition

\footnotetext{
${ }^{2}$ The variational inequality problem $V I(X, F(x))$ is to find a vector $\bar{x} \in X$ such that $(y-\bar{x})^{T} F(\bar{x}) \geq 0$ for all $y \in X[50]$.
}

is quite straightforward, and only a quadratic item needs to be added to the objective function. However, the problem in our paper is more complicated. The producer and consumer cannot be determined until the market clearing. In the equivalent problem, two items are added in the objective function, which is not a simple extension of the result in [51].

\section{Distributed Algorithm For Equilibrium SEEKING}

In this section, we first propose a distributed algorithm based on Nesterov's methods to solve the problem (14), i.e., to solve the game (7). Then, we use the nonexpansive operator theory to prove the convergence of the proposed algorithm.

\section{A. Algorithm design}

Before giving the algorithm, we first define a matrix and a function. Define the matrix

$$
\Theta:=\left[\begin{array}{ccc}
\Gamma & 0 & -I_{n} \\
0 & \sigma_{z}^{-1} I_{n} & -L \\
-I_{n} & -L & \sigma_{\mu}^{-1} I_{n}
\end{array}\right]
$$

where the matrix $\Gamma=\operatorname{diag}\left(\gamma_{i}\right), \forall i \in \mathcal{N} \cdot \gamma_{i}, \sigma_{z}$ and $\sigma_{\mu}$ are constant to make $\Theta$ positive definite ${ }^{3}$.

Define the function

$$
H_{i}\left(p_{i}\right)=\frac{\partial \tilde{h}_{i}}{\partial p_{i}}\left(p_{i}\right)+\gamma_{i} p_{i}
$$

where $\tilde{h}_{i}\left(p_{i}\right)=h_{i}\left(p_{i}\right)+\frac{p_{i}^{2}}{2\left(a_{i}-a_{\Sigma}\right)}-\frac{D_{i}}{a_{i}-a_{\Sigma}} p_{i}$. We have the following result.

Lemma 4. The function $H_{i}\left(p_{i}\right)$ is strongly monotone. Moreover, its inverse function $H_{i}^{-1}\left(p_{i}\right)$ exists and is also strongly monotone.

Proof. Because $\tilde{h}_{i}\left(p_{i}\right)$ is strongly convex, there is a constant $\tilde{h}_{0}>0$ such that for any $p_{i, 1}, p_{i, 2}$

$$
\left(p_{i, 1}-p_{i, 2}\right)^{\mathrm{T}}\left(\nabla \tilde{h}_{i}\left(p_{i, 1}\right)-\nabla \tilde{h}_{i}\left(p_{i, 2}\right)\right) \geq \tilde{h}_{0}\left\|p_{i, 1}-p_{i, 2}\right\|_{2}^{2}
$$

Then, we have

$$
\begin{aligned}
\left(p_{i, 1}\right. & \left.-p_{i, 2}\right)^{\mathrm{T}}\left(H_{i}\left(p_{i, 1}\right)-H_{i}\left(p_{i, 2}\right)\right)=\gamma_{i}\left\|p_{i, 1}-p_{i, 2}\right\|_{2}^{2} \\
& +\left(p_{i, 1}-p_{i, 2}\right)^{\mathrm{T}}\left(\nabla \tilde{h}_{i}\left(p_{i, 1}\right)-\nabla \tilde{h}_{i}\left(p_{i, 2}\right)\right) \\
& \geq\left(\tilde{h}_{0}+\gamma_{i}\right)\left\|p_{i, 1}-p_{i, 2}\right\|_{2}^{2}
\end{aligned}
$$

This implies that $H_{i}\left(p_{i}\right)$ is strongly monotone. For a strongly monotone function, its inverse function is also strongly monotone. This completes the proof.

We propose the following algorithm, which is denoted by

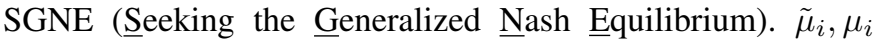
are variables of prosumer $i$ to estimate $\mu$. Because $\mu$ in the Lagrangian is a global variable, it needs to be estimated in a distributed way. Thus, we introduce $\tilde{\mu}_{i}, \mu_{i}$. Variables $\tilde{z}_{i}, z_{i}$ are introduced to force $\mu_{i}, i \in \mathcal{N}$ to reach consensus. The algorithm has the form of Nesterov's methods [38], [52]-[55], which consists of two phases: prediction phase and update

\footnotetext{
${ }^{3}$ It should be note that such $\gamma_{i}, \sigma_{z}, \sigma_{\mu}$ always exist to make $\Theta$ diagonally dominant, or positive definite.
} 


\section{Algorithm $1 S G N E$}

\section{Prediction phase:}

For prosumer $i$, it computes

$$
\begin{aligned}
\tilde{p}_{i, t} & =p_{i, t}+\eta\left(p_{i, t}-p_{i, t-1}\right) \\
\tilde{z}_{i, t} & =z_{i, t}+\eta\left(z_{i, t}-z_{i, t-1}\right) \\
\tilde{\mu}_{i, t} & =\mu_{i, t}+\eta\left(\mu_{i, t}-\mu_{i, t-1}\right)
\end{aligned}
$$

\section{Update phase:}

For prosumer $i$, it computes

$$
p_{i, t+1}=\left[H^{-1}\left(\gamma_{i} \tilde{p}_{i, t}-\tilde{\mu}_{i, t}\right)\right]_{\underline{p}_{i}}^{\bar{p}_{i}}
$$

Communicate with its neighbors $j \in N_{i}$ to get $\tilde{\mu}_{j, t}$, and compute

$$
z_{i, t+1}=\tilde{z}_{i, t}-\sigma_{z} \sum_{j \in N_{i}}\left(\tilde{\mu}_{i, t}-\tilde{\mu}_{j, t}\right)
$$

Communicate with its neighbors $j \in N_{i}$ to get $\tilde{z}_{j, t}, z_{j, t+1}$, and compute

$$
\begin{aligned}
& \mu_{i, t+1}=\tilde{\mu}_{i, t}+\sigma_{\mu}\left(2 p_{i, t+1}-\tilde{p}_{i, t}-D_{i}\right. \\
& \left.+2 \sum_{j \in N_{i}}\left(z_{i, t+1}-z_{j, t+1}\right)-\sum_{j \in N_{i}}\left(\tilde{z}_{i, t}-\tilde{z}_{j, t}\right)\right)
\end{aligned}
$$

phase. In the prediction phase, each bus uses the local stored information of the last two steps to get predictive variables by a linear extrapolation. In the update phase, the predictive variables are utilized to proceed the next iteration. In the algorithm, only communications with neighbors are needed, which means that it is fully distributed.

Compared with methods in [46], [47], the algorithm SGNE has two advantages. First, it does not need an extra variable to estimate the decision variables of other prosumers, which simplifies the algorithm by reducing $n-1$ order. This lightens the communication and computation burden. Second, each prosumer only needs to know its neighbors instead of the unified label of all prosumers. Then, if some prosumer joins or leaves, only its neighbors are affected. In contrast, all prosumers need to change the dimension of their state variables if methods in [46], [47] are adopted.

\section{B. Algorithm reformulation}

From (19d), we know

$$
H^{-1}\left(\gamma_{i} \tilde{p}_{i, t}-\tilde{\mu}_{i, t}\right)\left\{\begin{array}{l}
\leq p_{i, t+1}, p_{i, t+1}=\underline{p}_{i} \\
=p_{i, t+1}, \underline{p}_{i}<p_{i, t+1}<\bar{p}_{i} \\
\geq p_{i, t+1}, p_{i, t+1}=\bar{p}_{i}
\end{array}\right.
$$

Because $H_{i}^{-1}\left(p_{i}\right)$ is monotone, we have

$$
H\left(p_{i, t+1}\right)-\gamma_{i} \tilde{p}_{i, t}+\tilde{\mu}_{i, t}\left\{\begin{array}{l}
\geq 0, p_{i, t+1}=\underline{p}_{i} \\
=0, \underline{p}_{i}<p_{i, t+1}<\bar{p}_{i} \\
\leq 0, p_{i, t+1}=\bar{p}_{i}
\end{array}\right.
$$

It is equivalent to

$$
\begin{aligned}
p_{i, t+1}=\mathcal{P}_{\Omega_{i}}\left(p_{i, t+1}\right. & -\alpha_{p}\left(\nabla \tilde{h}_{i}\left(p_{i, t+1}\right)\right. \\
& \left.+\gamma_{i}\left(p_{i, t+1}-\tilde{p}_{i, t}\right)+\tilde{\mu}_{i, t}\right)
\end{aligned}
$$

Recalling $\mathcal{P}_{\Omega}(x)=\left(\mathrm{Id}+N_{\Omega}\right)^{-1}(x)$, we have

$$
\begin{aligned}
\gamma_{i}\left(\tilde{p}_{i, t}-p_{i, t+1}\right) & -\tilde{\mu}_{i, t}+\mu_{i, t+1} \in N_{\Omega_{i}}\left(p_{i, t+1}\right) \\
& +\nabla \tilde{h}_{i}\left(p_{i, t+1}\right)+\mu_{i, t+1}
\end{aligned}
$$

From (19e), we have

$$
\begin{aligned}
& \sigma_{z}^{-1}\left(\tilde{z}_{i, t}-z_{i, t+1}\right)-\sum_{j \in N_{i}}\left(\tilde{\mu}_{i, t}-\tilde{\mu}_{j, t}\right) \\
& \quad+\sum_{j \in N_{i}}\left(\mu_{i, t+1}-\mu_{j, t+1}\right)=\sum_{j \in N_{i}}\left(\mu_{i, t+1}-\mu_{j, t+1}\right)
\end{aligned}
$$

From (19f), we have

$$
\begin{aligned}
& \sigma_{\mu}^{-1}\left(\tilde{\mu}_{i, t}-\mu_{i, t+1}\right)+\sum_{j \in N_{i}}\left(z_{i, t+1}-z_{j, t+1}\right) \\
& -\sum_{j \in N_{i}}\left(\tilde{z}_{i, t}-\tilde{z}_{j, t}\right)+p_{i, t+1}-\tilde{p}_{i, t} \\
& =-p_{i, t+1}+D_{i}-\sum_{j \in N_{i}}\left(z_{i, t+1}-z_{j, t+1}\right)
\end{aligned}
$$

The compact form of the algorithm is

$$
\begin{aligned}
& \tilde{\boldsymbol{p}}_{t}=\boldsymbol{p}_{t}+\eta\left(\boldsymbol{p}_{t}-\boldsymbol{p}_{t-1}\right) \\
& \tilde{\boldsymbol{z}}_{t}=\boldsymbol{z}_{t}+\eta\left(\boldsymbol{z}_{t}-\boldsymbol{z}_{t-1}\right) \\
& \tilde{\boldsymbol{\mu}}_{t}=\boldsymbol{\mu}_{t}+\eta\left(\boldsymbol{\mu}_{t}-\boldsymbol{\mu}_{t-1}\right) \\
& \Gamma\left(\tilde{\boldsymbol{p}}_{t}-\boldsymbol{p}_{t+1}\right)-\left(\tilde{\boldsymbol{\mu}}_{t}-\boldsymbol{\mu}_{t+1}\right) \in N_{\Omega}\left(\boldsymbol{p}_{t+1}\right) \\
& +\nabla \tilde{h}\left(\boldsymbol{p}_{t+1}\right)+\boldsymbol{\mu}_{t+1} \\
& \sigma_{z}^{-1}\left(\tilde{\boldsymbol{z}}_{t}-\boldsymbol{z}_{t+1}\right)-L\left(\tilde{\boldsymbol{\mu}}_{t}-\boldsymbol{\mu}_{t+1}\right)=L \boldsymbol{\mu}_{t+1} \\
& \sigma_{\mu}^{-1}\left(\tilde{\boldsymbol{\mu}}_{t}-\boldsymbol{\mu}_{t+1}\right)-L\left(\tilde{z}_{t}-\boldsymbol{z}_{t+1}\right) \\
& -\left(\tilde{\boldsymbol{p}}_{t}-\boldsymbol{p}_{t+1}\right)=-\boldsymbol{p}_{t+1}+\boldsymbol{D}-L \boldsymbol{z}_{t+1}
\end{aligned}
$$

Define the following operator

$$
\mathcal{U}:\left[\begin{array}{c}
\boldsymbol{p} \\
\boldsymbol{z} \\
\boldsymbol{\mu}
\end{array}\right] \mapsto\left[\begin{array}{c}
\boldsymbol{\mu}+N_{\Omega}(\boldsymbol{p})+\nabla \tilde{h}(\boldsymbol{p}) \\
L \boldsymbol{\mu} \\
-\boldsymbol{p}+D-L \boldsymbol{z}
\end{array}\right]
$$

Define $\boldsymbol{\omega}_{t}=\operatorname{col}\left(\omega_{i, t}, i \in \mathcal{N}\right)=\operatorname{col}\left(p_{i, t}, z_{i, t}, \mu_{i, t}, i \in \mathcal{N}\right)$. Then, the algorithm (26) is rewritten as

$$
\begin{aligned}
& \tilde{\boldsymbol{\omega}}_{t}=\boldsymbol{\omega}_{t}+\eta\left(\boldsymbol{\omega}_{t}-\boldsymbol{\omega}_{t-1}\right) \\
& \Theta\left(\tilde{\boldsymbol{\omega}}_{t}-\boldsymbol{\omega}_{t+1}\right) \in \mathcal{U}\left(\boldsymbol{\omega}_{t+1}\right)
\end{aligned}
$$

where $\Theta$ is defined in (15). For the operator $\mathcal{U}$, it has the following properties

Lemma 5. Take step sizes $\Gamma, \alpha_{z}$ and $\alpha_{\mu}$ such that $\Theta$ is positive definite We have following properties.

1) Operator $\mathcal{U}$ is maximally monotone;

2) $\Theta^{-1} \mathcal{U}$ is maximally monotone under the $\Theta$-induced norm $\|\cdot\|_{\Theta}$

3) $\left(\operatorname{Id}+\Theta^{-1} \mathcal{U}\right)^{-1}$ exists and is firmly nonexpansive.

Proof. 1): The operator $\mathcal{U}$ can be rewritten as

$$
\begin{aligned}
\mathcal{U} & =\left[\begin{array}{ccc}
0 & 0 & I_{n} \\
0 & 0 & L \\
-I_{n} & -L & 0
\end{array}\right]\left[\begin{array}{c}
\boldsymbol{p} \\
\boldsymbol{z} \\
\boldsymbol{\mu}
\end{array}\right]+\left[\begin{array}{c}
N_{\Omega}(\boldsymbol{p})+\nabla \tilde{h}(\boldsymbol{p}) \\
\mathbf{0} \\
\boldsymbol{D}
\end{array}\right] \\
& =\mathcal{U}_{1}+\mathcal{U}_{2}
\end{aligned}
$$

As $\mathcal{U}_{1}$ is a skew-symmetric matrix, $\mathcal{U}_{1}$ is maximally monotone [48, Example 20.35]. Moreover, $N_{\Omega}(\boldsymbol{z})$ is maximally monotone by [48, Example 20.26]. In addition, $\boldsymbol{0}$ and $\boldsymbol{D}$ are monotone and continuous, which are all maximally monotone [48, Corollary 20.28]. As $\tilde{h}(\boldsymbol{p})$ is convex, $\nabla \tilde{h}(\boldsymbol{p})$ is also maximally monotone [48, Theorem 20.25]. Thus, $\mathcal{U}_{2}$ is also maximally monotone. Then, by [48, Corollary 25.5],we have $\mathcal{U}$ is maximally monotone.

2) As $\Theta$ is symmetric positive definite and $\mathcal{U}$ is maximally 
monotone, we can prove that $\Theta^{-1} \mathcal{U}$ is maximally monotone under the $\Theta$-induced norm by the similar analysis in Lemma 5.6 of [38].

3) As $\Theta^{-1} \mathcal{U}$ is maximally monotone, $\left(\operatorname{Id}+\Theta^{-1} \mathcal{U}\right)^{-1}$ exists and is firmly nonexpansive by [48, Proposition 23.8].

By the third assertion of Lemma 5, (28) is equivalent to

$$
\begin{aligned}
& \tilde{\boldsymbol{\omega}}_{t}=\boldsymbol{\omega}_{t}+\eta\left(\boldsymbol{\omega}_{t}-\boldsymbol{\omega}_{t-1}\right) \\
& \boldsymbol{\omega}_{t+1}=\left(\operatorname{Id}+\Theta^{-1} \mathcal{U}\right)^{-1} \tilde{\boldsymbol{\omega}}
\end{aligned}
$$

Up to now, we transform the algorithm SGNE to a fixed point problem with a nonexpansive operator $\left(\operatorname{Id}+\Theta^{-1} \mathcal{U}\right)^{-1}$, which provides fundamental support for the convergence proof.

\section{NASh EQuilibrium Seeking AND Convergence}

In this section, we address the optimality of the equilibrium point and the convergence of the algorithm SGNE, i.e., the discrete dynamic system (19).

\section{A. Nash Equilibrium}

First, we define the equilibrium of the algorithm SGNE.

Definition 1. A point $\boldsymbol{\omega}^{*}=\operatorname{col}\left(\omega_{i}^{*}, \quad i \in \mathcal{N}\right)=$ $\operatorname{col}\left(p_{i}^{*}, z_{i}^{*}, \mu_{i}^{*}, i \in \mathcal{N}\right)$ is an equilibrium point of (19) if $\lim _{t \rightarrow+\infty} \omega_{i, t}=\omega_{i}^{*}$ holds for all $i$.

Recalling the (B.5), and we have the following result.

Theorem 6. Suppose assumptions 1,2 and 3 hold. At the equilibrium of the algorithm SGNE, we have $\mu_{i}^{*}=\mu_{j}^{*}=$ $\mu_{0}, \forall i, j$ is the clearing price and $\left(\boldsymbol{p}^{*}, \boldsymbol{b}^{*}\right)$ is the GNE of the game, where $\mu_{0}$ is constant.

Proof. From Definition 1 and (26), we have

$$
\begin{aligned}
& \mathbf{0} \in N_{\Omega}\left(\boldsymbol{p}^{*}\right)+\nabla \tilde{h}\left(\boldsymbol{p}^{*}\right)+\boldsymbol{\mu}^{*} \\
& \mathbf{0}=L \boldsymbol{\mu}^{*} \\
& \mathbf{0}=-\boldsymbol{p}^{*}+\boldsymbol{D}-L \boldsymbol{z}^{*}
\end{aligned}
$$

From (31b), we have

$$
\mu_{i}^{*}=\mu_{j}^{*}=\mu_{0}, \forall i, j
$$

From (31c), we have

$$
0=-\mathbf{1}^{\mathrm{T}} \boldsymbol{p}^{*}+\mathbf{1}^{\mathrm{T}} \boldsymbol{D}-\mathbf{1}^{\mathrm{T}} L \boldsymbol{z}^{*}=-\mathbf{1}^{\mathrm{T}} \boldsymbol{p}^{*}+\mathbf{1}^{\mathrm{T}} \boldsymbol{D}
$$

The equations (31a), (32) and (33) are the KKT condition (B.7). This completes the proof.

\section{B. Convergence}

In this subsection, we analyze the convergence of the algorithm SGNE based on the compact form (30). First, we give the following result.

Theorem 7. Suppose Assumption 1 and Assumption 2 hold. Given a parameter $\eta$ satisfying $0<\eta<\frac{1}{3}$ and the step sizes $\Gamma, \alpha_{z}$ and $\alpha_{\mu}$ such that $\Theta$ is positive definite. Then with SGNE, $\boldsymbol{\omega}_{t}$ converges to a primal-dual optimal solution $\boldsymbol{\omega}^{*}$ of the problem (14). Then, $\left(\boldsymbol{p}^{*}, \boldsymbol{b}^{*}\right)$ is the GNE of (7).

The detailed proof of Theorem 7 is given in Appendix C. Here we introduce the outline of the proof. Frist, we prove that $\lim _{t \rightarrow \infty}\left(\boldsymbol{\omega}_{t+1}-\boldsymbol{\omega}_{t}\right)=0$. Then, we prove that

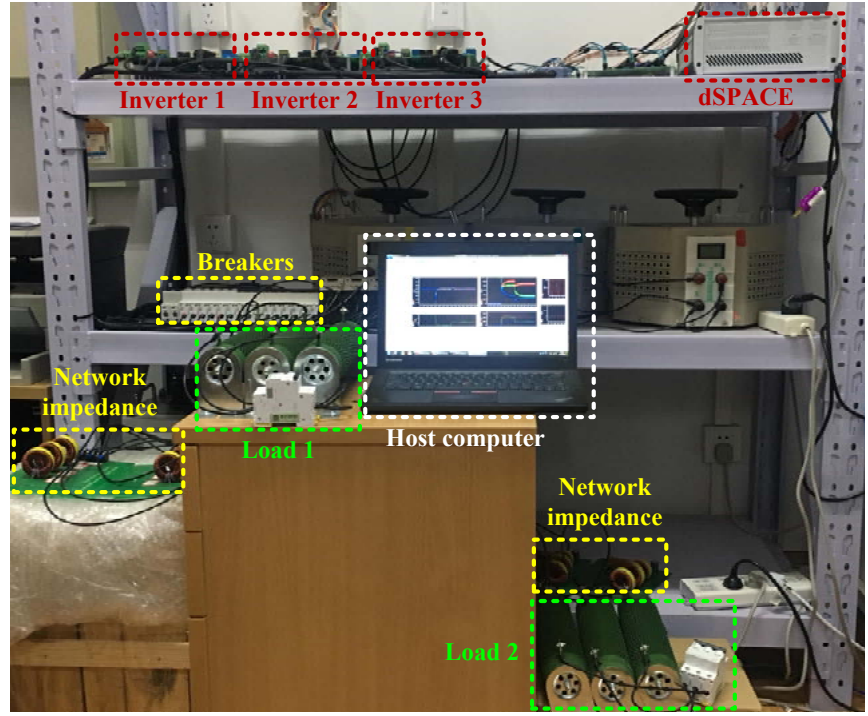

Fig. 1. Experiment platform based on dSPACE RTI 1202 controller

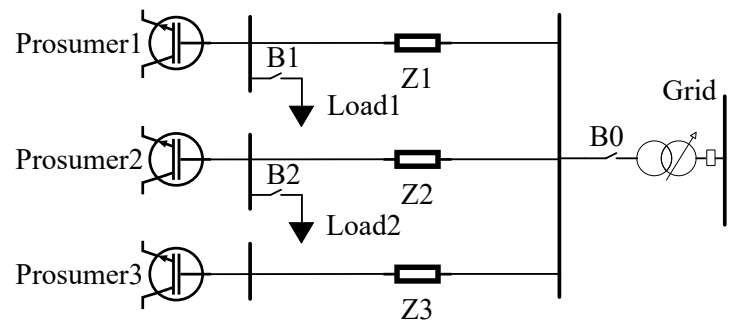

Fig. 2. Topology of the experiment system

$\left\|\boldsymbol{\omega}_{t}-\boldsymbol{\omega}^{*}\right\|_{\Theta}^{2}$ converges to some constant. Finally, we prove that $\left\|\boldsymbol{\omega}_{t}-\boldsymbol{\omega}^{*}\right\|_{\Theta}^{2}$ converges to zero.

Invoking Theorem 3, if Assumption 3 also holds, the algorithm will converge to the unique GNE of the GNG (7), equivalently the energy sharing game (5).

\section{EXPERIMENTS AND SimUlation STUdies}

In this section, experiments and numerical simulations are introduced to verify the effectiveness of the proposed method. First, experiments with three prosumers are carried out to illustrate the basic properties of the algorithm. Then, a case with 1888 prosumers is investigated to test the scalability, where the communication topology is identical to the topology of the IEEE 1888-bus system [56].

\section{A. Experimental results}

The proposed method is verified on an experimental platform based on the dSPACE RTI 1202 controller, which is presented in Fig.1. It is composed of three inverters, one dSPACE RTI 1202 controller, two switchable loads, and one host computer. Each inverter represents a prosumer, which can both produce and consume power. The system topology is given in Fig.2. In the experiments, the breaker B0 is open, i.e., the system operates in an isolated mode. One load is connected at the bus of Prosumer1 and the other is at the bus of Prosumer2. Three prosumers are connected through impedances. The communication topology is Prosumer $1 \leftrightarrow$ Prosumer $2 \leftrightarrow$ Prosumer $3 \leftrightarrow$ Prosumer1. The disutility function is $h_{i}\left(p_{i}\right)=\frac{1}{2} c_{i} p_{i}^{2}+d_{i} p_{i}$ with $c_{1}=0.00075, c_{2}=$ 


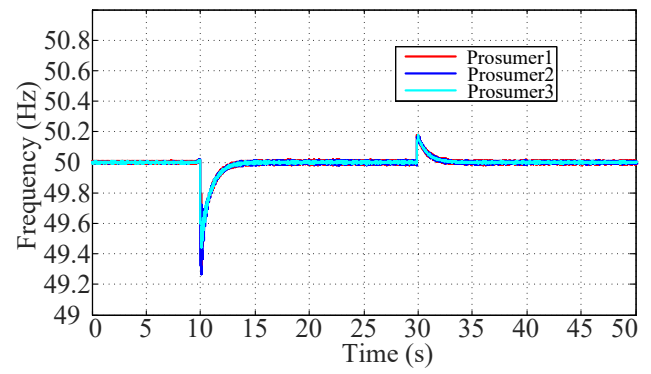

Fig. 3. The frequency dynamics of three DGs

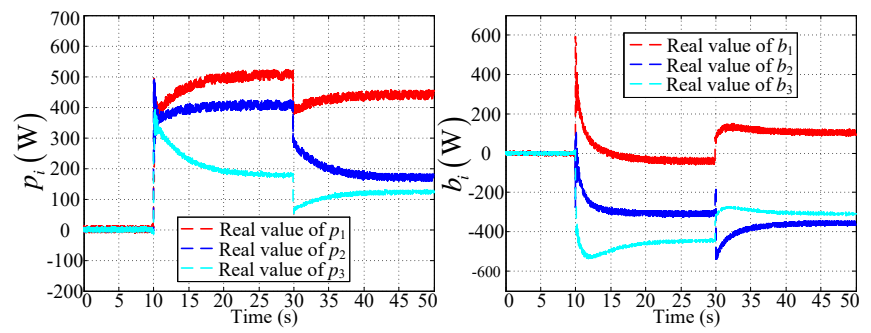

Fig. 4. Dynamics of the GNE

$0.0006, c_{3}=0.001, d_{i}=0$. The price elasticity of each prosumer is set as $a_{i}=-1000$. The load demand of each prosumer is $D_{1}=730 \mathrm{~W}, D_{2}=365 \mathrm{~W}, D_{3}=0$.

The simulation scenario is: 1) at $t=10 \mathrm{~s}$, two loads are connected; 2) at $t=30 \mathrm{~s}$, load 2 is disconnected. Then, each DG regulates its generation to balance the power difference. The frequency dynamics are illustrated in Fig.3. When loads are connected, the frequency drops to about $49.2 \mathrm{~Hz}$ and recovers to the nominal value in four seconds. On the contrary, when load 2 is switched off, the frequency increases and recovers in 3 seconds.

The GNE of the first stage is $\left(p_{1}^{*}=505.4 \mathrm{~W}, b_{1}^{*}=\right.$ $103.3 \mathrm{~W}),\left(p_{2}^{*}=408.4 \mathrm{~W}, b_{2}^{*}=355.0 \mathrm{~W}\right),\left(p_{3}^{*}=177.8 \mathrm{~W}, b_{3}^{*}=\right.$ $310.0 \mathrm{~W})$. Dynamics of seeking the GNE is illustrated in Fig.4, where the left part is the generation $p_{i}$ of each prosumer and the right part is the purchase willingness $b_{i}$. They vary slightly around the equilibrium. In this stage, Prosumer1 buys power from Prosumer2 and Prosumer3. The GNE of the second stage is $\left(p_{1}^{*}=440.6 \mathrm{~W}, b_{1}^{*}=-41.7 \mathrm{~W}\right),\left(p_{2}^{*}=168.9 \mathrm{~W}, b_{2}^{*}=\right.$ $-310.0 \mathrm{~W}),\left(p_{3}^{*}=123.9 \mathrm{~W}, b_{3}^{*}=-444.2 \mathrm{~W}\right)$. Compared with the results obtained from the centralized method, the steady state generations are optimal to the problem (14). This shows that the GNE is obtained. Moreover, real power can always trace the reference value. This verifies that the proposed method can get the correct results in the experiment. Dynamics of the payoff functions are given in Fig.5, which also converges in five seconds. In the first stage, Prosumer 3 earns profit by selling power to Prosumer1 and Prosumer2. In the second stage, there is only Prosumer1 buying power, while others selling. The cost of Prosumer 2 changes from positive to negative, which implies that it earns profit by selling power to Prosumer1.

\section{B. Scalability}

In this subsection, scalability of the proposed method is illustrated by using 1888 prosumers. The communication topology is the same with the physical topology of case1888rte

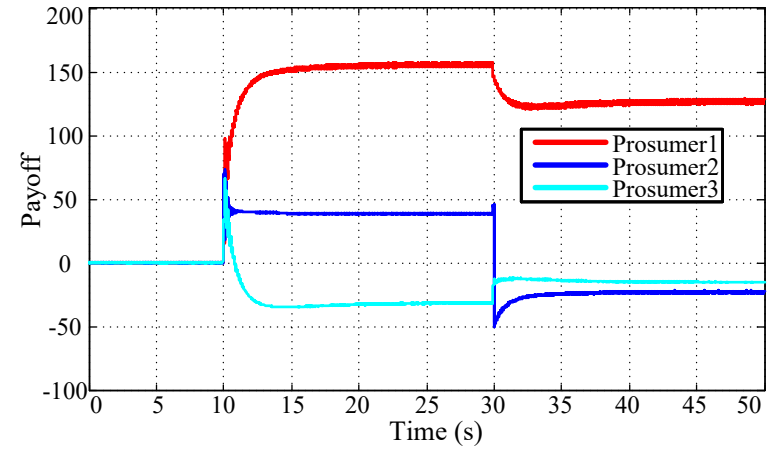

Fig. 5. Dynamics of the payoff functions

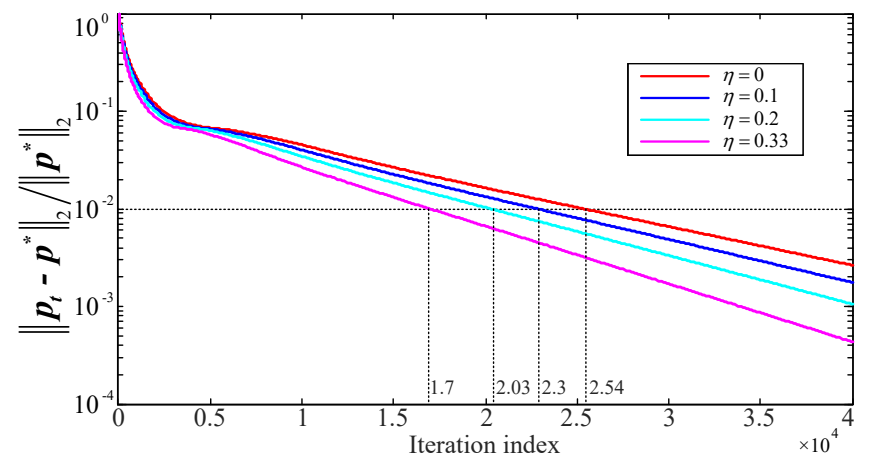

Fig. 6. Error of 1888 prosumers with different $\eta$

system in Matpower [57]. The 1888 prosumers is numbered with the same number of case1888rte system. $c_{i}$ in the disutility function is $c_{i}=10+10 \times \frac{i}{1888}$ and $d_{i}=10 . a_{i}$ of these 1888 prosumers are all -1 . The effect of $\eta$ is also investigated, which is set as $0,0.1,0.2,0.33$ respectively. The convergence of $p_{i, t}$ to the equilibrium point is given in Fig.6. With $\eta=0$, it takes more than 25,400 iterations to make the relative error smaller than $1 \%$. With $\eta=0.33$, it takes about 17,000 iterations, which is reduced by $33 \%$. It is also observed that a larger $\eta$ results in a higher convergence rate.

\section{Comparison with existing methods}

To further verify the performance of the proposed algorithm, comparisons with distributed ADMM and distributed gradient descent method are investigated with 1888 prosumers. The ADMM can refer to [58]-[60]. The distributed gradient descent algorithm is explained in [61], [62]. The simulation scenario is the same as that in Section VII.B. The result is illustrated in Fig.7. With distributed ADMM, it takes more than 22, 500 iterations to make the relative error smaller than $1 \%$. With distributed gradient descent algorithm, it takes about 25,300 iterations. They are both inferior to the result of SGNE with $\eta=0.33$, which takes 17,000 iterations. If we further reduce the error to $0.1 \%$, the performance of SGNE is much better. It takes 34,000 iterations, while the distributed gradient descent method takes 51,000 iterations. The ADMM fails to reduce the error smaller than $0.1 \%$ within 60,000 iterations. This validates the performance of the proposed method.

\section{CONCLUSION}

In this work, the energy sharing game among prosumers is formulated, whose objective function of each prosumer is 


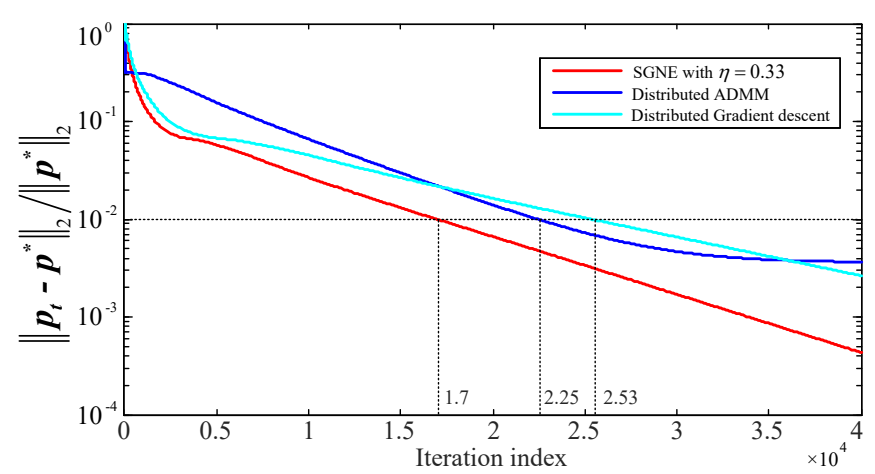

Fig. 7. Comparison with distributed ADMM and distributed gradient descent algorithms.

associated with the decisions of all the prosumers. To solve it in a fully distributed way, the game is transformed into an equivalent optimization problem. Then, an algorithm based on Nesterov's methods is devised to solve the problem and consequently find the GNE in a fully distributed manner with neighboring communication. The convergence of the algorithm is proved rigorously. Experimental results with three prosumers show that the GNE can be obtained at a fast speed. Simulations on 1888 prosumers verify the scalability of the proposed method.

This paper offers a different perspective for seeking the GNE in the GNG. If the objective function of one prosumer is associated with too many other prosumers, it is possible to transform the GNG to an equivalent optimization problem to avoid dense communication. In the future work, it is interesting to investigate the conditions of the equivalent transformation for a more general GNG. Moreover, noise may exist in the system [63], and it is also very important to study robust algorithms in the future.

\section{APPENDIX A PROOFS OF THEOREM 1}

Proof. For the first part of $f_{i}\left(p_{i}, \boldsymbol{b}\right)$, because $h_{i}\left(p_{i}\right)$ is strictly convex and only determined by prosumer $i$, its gradient and pseudo-gradient are the same and strictly monotone.

For the second part of $f_{i}\left(p_{i}, \boldsymbol{b}\right)$, the pseudo-gradient of $g_{i}(\boldsymbol{b}), i \in \mathcal{N}$ is

$$
\begin{aligned}
G(\boldsymbol{b}) & =\operatorname{col}\left(\nabla_{b_{1}} g_{1}(\boldsymbol{b}), \cdots, \nabla_{b_{N}} g_{N}(\boldsymbol{b})\right) \\
& =\operatorname{col}\left(2 \alpha_{i} b_{i}+\beta_{i} \sum_{j \neq i} b_{j}\right) \\
& =A \boldsymbol{b}
\end{aligned}
$$

where

$$
[A]_{i j}= \begin{cases}2 \alpha_{i}=2 \frac{a_{i}-a_{\Sigma}}{a_{\Sigma}^{2}}, & j=i \\ \beta_{i}=\frac{2 a_{i}-a_{\Sigma}}{a_{\Sigma}^{2}}, & j \neq i\end{cases}
$$

We need to prove that, for $\forall \boldsymbol{x}, \boldsymbol{y} \in \mathbb{R}^{n}$, there exists a constant $\rho_{0}>0$ such that

$$
\begin{aligned}
(\boldsymbol{x}-\boldsymbol{y})^{\mathrm{T}} A^{\mathrm{T}}(\boldsymbol{x}-\boldsymbol{y}) & =\frac{1}{2}(\boldsymbol{x}-\boldsymbol{y})^{\mathrm{T}}\left(A^{\mathrm{T}}+A\right)(\boldsymbol{x}-\boldsymbol{y}) \\
& \geq \rho_{0}\|\boldsymbol{x}-\boldsymbol{y}\|^{2}
\end{aligned}
$$

It suffices to prove that $A^{\mathrm{T}}+A$ is positive definite.
Define the matrix $\hat{A}$ with elements as

$$
[\hat{A}]_{i j}= \begin{cases}-2\left(a_{\Sigma}-a_{i}\right), & j=i \\ -a_{\Sigma}+a_{i}+a_{j}, & j \neq i\end{cases}
$$

Then the matrix $\frac{1}{2}\left(A^{\mathrm{T}}+A\right)=\frac{1}{a_{\Sigma}^{2}} \hat{A}$. Next, we will prove that $\hat{A}$ is positive definite. Recall that $I_{n}$ is an identity matrix, and $E_{n}$ is a matrix with all of elements as 1 . Then, matrix $\hat{A}$ can be divided by

$$
\hat{A}=-a_{\Sigma} \cdot I_{n}-a_{\Sigma} \cdot E_{n}+\operatorname{diag}\left(a_{i}\right) \cdot E_{n}+E_{n} \cdot \operatorname{diag}\left(a_{i}\right)
$$

Take any $\boldsymbol{x} \in \mathbb{R}^{n}, \boldsymbol{x} \neq \mathbf{0}$ with elements $x_{i}$, and we have

$$
\begin{aligned}
& \boldsymbol{x}^{T} \hat{A} \boldsymbol{x}=-\boldsymbol{x}^{T}\left(a_{\Sigma} \cdot I_{n}+a_{\Sigma} \cdot E_{n}\right) \boldsymbol{x} \\
& +\boldsymbol{x}^{T}\left(\operatorname{diag}\left(a_{i}\right) \cdot E_{n}+E_{n} \cdot \operatorname{diag}\left(a_{i}\right)\right) \boldsymbol{x} \\
& =-a_{\Sigma} \cdot \boldsymbol{x}^{T}\left(I_{n}+E_{n}\right) \boldsymbol{x}+2\left(\sum_{i=1}^{n} a_{i} x_{i}\right)\left(\sum_{i=1}^{n} x_{i}\right) \\
& =-a_{\Sigma}\left(\sum_{i=1}^{n} x_{i}^{2}+\left(\sum_{i=1}^{n} x_{i}\right)^{2}\right) \\
& +2\left(\sum_{i=1}^{n} a_{i} x_{i}\right)\left(\sum_{i=1}^{n} x_{i}\right) \\
& \geq-a_{\Sigma}\left(\sum_{i=1}^{n} x_{i}^{2}+\left(\sum_{i=1}^{n} x_{i}\right)^{2}\right) \\
& -2\left|\sum_{i=1}^{n} a_{i} x_{i}\right| \cdot\left|\sum_{i=1}^{n} x_{i}\right| \\
& >\sqrt{\sum_{i=1}^{n} a_{i}^{2}}\left(\sum_{i=1}^{n} x_{i}^{2}+\left(\sum_{i=1}^{n} x_{i}\right)^{2}\right) \\
& -2\left|\sum_{i=1}^{n} a_{i} x_{i}\right| \cdot\left|\sum_{i=1}^{n} x_{i}\right| \\
& \geq \sqrt{\sum_{i=1}^{n} a_{i}^{2}}\left(\sum_{i=1}^{n} x_{i}^{2}+\left(\sum_{i=1}^{n} x_{i}\right)^{2}\right) \\
& -2 \sqrt{\left(\sum_{i=1}^{n} a_{i}^{2}\right) \cdot\left(\sum_{i=1}^{n} x_{i}^{2}\right)} \cdot\left|\sum_{i=1}^{n} x_{i}\right| \\
& =\sqrt{\sum_{i=1}^{n} a_{i}^{2}}\left(\sqrt{\sum_{i=1}^{n} x_{i}^{2}}-\left|\sum_{i=1}^{n} x_{i}\right|\right)^{2} \\
& \geq 0
\end{aligned}
$$

where the first ' $\geq$ ' is due to

$$
\left|\sum_{i=1}^{n} a_{i} x_{i}\right| \cdot\left|\sum_{i=1}^{n} x_{i}\right| \geq\left(\sum_{i=1}^{n} a_{i} x_{i}\right) \cdot\left(\sum_{i=1}^{n} x_{i}\right),
$$

the ' $>$ ' is due to $a_{i}<0, \forall i$, and

$$
\left(a_{1}+a_{2}+\cdots+a_{n}\right)^{2} \geq a_{1}^{2}+a_{2}^{2}+\cdots+a_{n}^{2},
$$

and the second ' $\geq$ ' can be obtained from the Cauchy-Schwarz inequality, i.e.,

$$
\left(\sum_{i=1}^{n} a_{i} x_{i}\right)^{2} \leq\left(\sum_{i=1}^{n} a_{i}^{2}\right) \cdot\left(\sum_{i=1}^{n} x_{i}^{2}\right)
$$

Thus, for any $\boldsymbol{x} \in \mathbb{R}^{n}, \boldsymbol{x} \neq \mathbf{0}$ with elements $x_{i}$, we have $\boldsymbol{x}^{T} \hat{A} \boldsymbol{x}>0$. This implies that $\frac{1}{2}\left(A^{\mathrm{T}}+A\right)$ is positive definite, i.e., $G(\boldsymbol{b})$ is strongly monotone. This completes the proof. 


\section{APPENDIX B \\ PROOFS OF THEOREM 3}

Proof. $\Rightarrow$ 1) From Lemma 2, we need to find $\boldsymbol{x}^{*}:=\left(p_{i}^{*}, b_{i}^{*}\right) \in$ $X$ such that

$$
\left\langle F\left(\boldsymbol{x}^{*}\right), \boldsymbol{x}-\boldsymbol{x}^{*}\right\rangle \geq 0, \forall \boldsymbol{x} \in X
$$

Now, we check the KKT condition for $\operatorname{VI}(X, F(\boldsymbol{p}, \boldsymbol{b}))$ in (B.1). In fact, $\boldsymbol{x}^{*}$ is a solution to $\operatorname{VI}(X, F(\boldsymbol{p}, \boldsymbol{b}))$ in (B.1) if and only if $\boldsymbol{x}^{*}$ is the optimal solution to the following optimization problem:

$$
\min _{\boldsymbol{x} \in \mathbb{R}^{2 n}}\left\langle F\left(\boldsymbol{x}^{*}\right), \boldsymbol{x}\right\rangle, \quad \text { s.t. } \boldsymbol{x} \in X
$$

If $\boldsymbol{x}^{*}$ solves (B.2), there exists $\boldsymbol{\lambda}^{*} \in \mathbb{R}$ such that the following optimality conditions (KKT) are satisfied [64, Theorem 3.25]

$$
\begin{aligned}
& 0 \in h_{i}^{\prime}\left(p_{i}^{*}\right)+\lambda_{i}^{*}+N_{\Omega_{i}}\left(p_{i}^{*}\right) \\
& 0=-\left(2 a_{i} \mu_{c}+b_{i}^{*}+a_{i} \lambda_{i}^{*}\right) \frac{1}{a_{\Sigma}}+\mu_{c}+\lambda_{i}^{*} \\
& 0=p_{i}^{*}+a_{i} \mu_{c}^{*}+b_{i}^{*}-D_{i} \\
& 0=a_{\Sigma} \mu_{c}^{*}+b_{i}^{*}+\sum_{j \neq i} b_{j}^{*}
\end{aligned}
$$

From (B.3b), we know

$$
\begin{gathered}
0=-\left(2 a_{i} \mu_{c}^{*}+b_{i}^{*}+a_{i} \lambda_{i}^{*}\right) \frac{1}{a_{\Sigma}}+\mu_{c}^{*}+\lambda_{i}^{*} \\
\Rightarrow \lambda_{i}^{*}=\frac{a_{\Sigma}-2 a_{i}}{a_{i}-a_{\Sigma}} \mu_{c}^{*}-\frac{1}{a_{i}-a_{\Sigma}} b_{i}^{*}
\end{gathered}
$$

From (B.3c), we know

$$
b_{i}^{*}=D_{i}-p_{i}^{*}-a_{i} \mu_{c}^{*}
$$

Combining (B.4) and (B.5), we have

$$
\begin{aligned}
\lambda_{i}^{*} & =\frac{a_{\Sigma}-2 a_{i}}{a_{i}-a_{\Sigma}} \mu_{c}^{*}-\frac{D_{i}-p_{i}^{*}-a_{i} \mu_{c}^{*}}{a_{i}-a_{\Sigma}} \\
& =-\mu_{c}^{*}+\frac{p_{i}^{*}}{a_{i}-a_{\Sigma}}-\frac{D_{i}}{a_{i}-a_{\Sigma}}
\end{aligned}
$$

Then, the KKT condition (B.3) is

$$
\begin{aligned}
& 0 \in h_{i}^{\prime}\left(p_{i}^{*}\right)+\frac{p_{i}^{*}}{a_{i}-a_{\Sigma}}-\frac{D_{i}}{a_{i}-a_{\Sigma}}-\mu_{c}^{*}+N_{\Omega_{i}}\left(p_{i}^{*}\right) \\
& 0=\sum_{i \in \mathcal{N}} D_{i}-\sum_{i \in \mathcal{N}} p_{i}
\end{aligned}
$$

It is also the KKT condition of the following problem

$$
\begin{array}{ll}
\min _{\boldsymbol{p}} & \tilde{h}(\boldsymbol{p})=\sum_{i \in \mathcal{N}}\left(h_{i}\left(p_{i}\right)+\frac{p_{i}^{2}}{2\left(a_{i}-a_{\Sigma}\right)}-\frac{D_{i}}{a_{i}-a_{\Sigma}} p_{i}\right) \\
\text { s.t. } \quad \sum_{i \in \mathcal{N}} p_{i}=\sum_{i \in \mathcal{N}} D_{i}, \quad \mu_{c} \\
\quad \underline{p}_{i} \leq p_{i} \leq \bar{p}_{i}
\end{array}
$$

where $\mu_{c}$ is the Lagrangian multiplier.

Define

$$
\tilde{h}_{i}\left(p_{i}\right)=h_{i}\left(p_{i}\right)+\frac{p_{i}^{2}}{2\left(a_{i}-a_{\Sigma}\right)}-\frac{D_{i}}{a_{i}-a_{\Sigma}} p_{i}
$$

We know $\tilde{h}_{i}\left(p_{i}\right)$ is strongly convex and the Slater's condition holds by Assumption 2. Thus, $p_{i}^{*}$ and $\mu_{c}^{*}$ exist. By (B.5), $b_{i}^{*}$ also exists.

$\Rightarrow 2$ ) If Assumption 3 holds, there exists at least one prosumer $i$ with $\underline{p}_{i}<p_{i}^{*}<\bar{p}_{i}$. For this $i$, we have
$\{0\}=N_{\Omega_{i}}\left(p_{i}^{*}\right)$. Then, by (B.7a), $\mu_{c}^{*}$ is unique. The unique $b_{i}^{*}$ can be obtained from (B.5).

\section{APPENDIX C}

PROOFS OF THEOREM 7

Proof. Frist, we prove that $\lim _{t \rightarrow \infty}\left(\boldsymbol{\omega}_{t+1}-\boldsymbol{\omega}_{t}\right)=0$. Given any equilibrium point $\omega^{*}$, use the equation (1), and we have

$$
\begin{aligned}
\| \boldsymbol{\omega}_{t}- & \boldsymbol{\omega}^{*}\left\|_{\Theta}^{2}-\right\| \boldsymbol{\omega}_{t+1}-\boldsymbol{\omega}^{*} \|_{\Theta}^{2} \\
= & 2\left\langle\boldsymbol{\omega}_{t}-\boldsymbol{\omega}_{t+1}, \boldsymbol{\omega}_{t}-\boldsymbol{\omega}^{*}\right\rangle_{\Theta}-\left\|\boldsymbol{\omega}_{t}-\boldsymbol{\omega}_{t+1}\right\|_{\Theta}^{2} \\
= & 2\left\langle\boldsymbol{\omega}_{t}-\boldsymbol{\omega}_{t+1}, \boldsymbol{\omega}_{t+1}-\boldsymbol{\omega}^{*}\right\rangle_{\Theta}+\left\|\boldsymbol{\omega}_{t}-\boldsymbol{\omega}_{t+1}\right\|_{\Theta}^{2} \\
= & \left\|\boldsymbol{\omega}_{t}-\boldsymbol{\omega}_{t+1}\right\|_{\Theta}^{2}+2\left\langle\tilde{\boldsymbol{\omega}}_{t}-\boldsymbol{\omega}_{t+1}, \boldsymbol{\omega}_{t+1}-\boldsymbol{\omega}^{*}\right\rangle_{\Theta} \\
& \quad-2 \eta\left\langle\boldsymbol{\omega}_{t}-\boldsymbol{\omega}_{t-1}, \boldsymbol{\omega}_{t+1}-\boldsymbol{\omega}^{*}\right\rangle_{\Theta}
\end{aligned}
$$

where the last equation is derived by integrating (30a).

By (28b), we have

$$
0 \in \mathcal{U}\left(\omega^{*}\right)
$$

Since $\mathcal{U}$ is maximally monotone, we have

$$
\left\langle\Theta\left(\tilde{\boldsymbol{\omega}}_{t}-\boldsymbol{\omega}_{t+1}\right), \boldsymbol{\omega}_{t+1}-\boldsymbol{\omega}^{*}\right\rangle \geq 0
$$

Thus, we have

$$
\begin{aligned}
& \left\|\boldsymbol{\omega}_{t+1}-\boldsymbol{\omega}^{*}\right\|_{\Theta}^{2}-\left\|\boldsymbol{\omega}_{t}-\boldsymbol{\omega}^{*}\right\|_{\Theta}^{2} \\
& \quad \leq 2 \eta\left\langle\boldsymbol{\omega}_{t}-\boldsymbol{\omega}_{t-1}, \boldsymbol{\omega}_{t+1}-\boldsymbol{\omega}^{*}\right\rangle_{\Theta}-\left\|\boldsymbol{\omega}_{t}-\boldsymbol{\omega}_{t+1}\right\|_{\Theta}^{2}
\end{aligned}
$$

Moreover,

$$
\begin{aligned}
& \eta\left(\left\|\boldsymbol{\omega}_{t}-\boldsymbol{\omega}^{*}\right\|_{\Theta}^{2}-\left\|\boldsymbol{\omega}_{t-1}-\boldsymbol{\omega}^{*}\right\|_{\Theta}^{2}\right) \\
& \quad=\eta\left(2\left\langle\boldsymbol{\omega}_{t}-\boldsymbol{\omega}_{t-1}, \boldsymbol{\omega}_{t}-\boldsymbol{\omega}^{*}\right\rangle_{\Theta}-\left\|\boldsymbol{\omega}_{t}-\boldsymbol{\omega}_{t-1}\right\|_{\Theta}^{2}\right)
\end{aligned}
$$

From (C.4) and (C.5), we have

$$
\begin{gathered}
\left\|\boldsymbol{\omega}_{t+1}-\boldsymbol{\omega}^{*}\right\|_{\Theta}^{2}-\left\|\boldsymbol{\omega}_{t}-\boldsymbol{\omega}^{*}\right\|_{\Theta}^{2} \\
\quad-\eta\left(\left\|\boldsymbol{\omega}_{t}-\boldsymbol{\omega}^{*}\right\|_{\Theta}^{2}-\left\|\boldsymbol{\omega}_{t-1}-\boldsymbol{\omega}^{*}\right\|_{\Theta}^{2}\right) \\
\leq 2 \eta\left\langle\boldsymbol{\omega}_{t}-\boldsymbol{\omega}_{t-1}, \boldsymbol{\omega}_{t+1}-\boldsymbol{\omega}^{*}\right\rangle_{\Theta}-\left\|\boldsymbol{\omega}_{t}-\boldsymbol{\omega}_{t+1}\right\|_{\Theta}^{2} \\
\quad-\eta\left(2\left\langle\boldsymbol{\omega}_{t}-\boldsymbol{\omega}_{t-1}, \boldsymbol{\omega}_{t}-\boldsymbol{\omega}^{*}\right\rangle_{\Theta}-\left\|\boldsymbol{\omega}_{t}-\boldsymbol{\omega}_{t-1}\right\|_{\Theta}^{2}\right) \\
=-\left\|\boldsymbol{\omega}_{t}-\boldsymbol{\omega}_{t+1}\right\|_{\Theta}^{2}+2 \eta\left\langle\boldsymbol{\omega}_{t}-\boldsymbol{\omega}_{t-1}, \boldsymbol{\omega}_{t+1}-\boldsymbol{\omega}_{t}\right\rangle_{\Theta} \\
\quad+\eta\left\|\boldsymbol{\omega}_{t}-\boldsymbol{\omega}_{t-1}\right\|_{\Theta}^{2} \\
\leq(\eta-1)\left\|\boldsymbol{\omega}_{t}-\boldsymbol{\omega}_{t+1}\right\|_{\Theta}^{2}+2 \eta\left\|\boldsymbol{\omega}_{t}-\boldsymbol{\omega}_{t-1}\right\|_{\Theta}^{2}
\end{gathered}
$$

Define a sequence $s_{k}=\left\|\boldsymbol{\omega}_{t}-\boldsymbol{\omega}^{*}\right\|_{\Theta}^{2}-\eta\left\|\boldsymbol{\omega}_{t-1}-\boldsymbol{\omega}^{*}\right\|_{\Theta}^{2}+$ $2 \eta\left\|\boldsymbol{\omega}_{t}-\boldsymbol{\omega}_{t-1}\right\|_{\Theta}^{2}$. Then

$$
\begin{aligned}
s_{k+1}-s_{k}= & \left\|\boldsymbol{\omega}_{t+1}-\boldsymbol{\omega}^{*}\right\|_{\Theta}^{2}-\left\|\boldsymbol{\omega}_{t}-\boldsymbol{\omega}^{*}\right\|_{\Theta}^{2} \\
& -\eta\left(\left\|\boldsymbol{\omega}_{t}-\boldsymbol{\omega}^{*}\right\|_{\Theta}^{2}-\left\|\boldsymbol{\omega}_{t-1}-\boldsymbol{\omega}^{*}\right\|_{\Theta}^{2}\right) \\
& +2 \eta\left\|\boldsymbol{\omega}_{t+1}-\boldsymbol{\omega}_{t}\right\|_{\Theta}^{2}-2 \eta\left\|\boldsymbol{\omega}_{t}-\boldsymbol{\omega}_{t-1}\right\|_{\Theta}^{2} \\
\leq & (3 \eta-1)\left\|\boldsymbol{\omega}_{t+1}-\boldsymbol{\omega}_{t}\right\|_{\Theta}^{2}
\end{aligned}
$$

From (C.7), we have $(1-3 \eta) \sum_{t=1}^{k}\left\|\boldsymbol{\omega}_{t+1}-\boldsymbol{\omega}_{t}\right\|_{\Theta}^{2} \leq s_{1}-$ $s_{k+1} \leq s_{1}+\eta\left\|\boldsymbol{\omega}_{t}-\boldsymbol{\omega}^{*}\right\|_{\Theta}^{2}$. As $0<\eta<\frac{1}{3}$, we have $s_{k+1} \leq$ $s_{k} \leq s_{1}$ and $\left\|\boldsymbol{\omega}_{t}-\boldsymbol{\omega}^{*}\right\|_{\Theta}^{2}-\eta\left\|\boldsymbol{\omega}_{t-1}-\boldsymbol{\omega}^{*}\right\|_{\Theta}^{2} \leq s_{k} \leq s_{1}$. Then, $\left\|\boldsymbol{\omega}_{t}-\boldsymbol{\omega}^{*}\right\|_{\Theta}^{2} \leq \eta^{k}\left(\left\|\boldsymbol{\omega}_{1}-\boldsymbol{\omega}^{*}\right\|_{\Theta}^{2}-\frac{\mu_{1}}{1-\alpha}\right)+\frac{\mu_{1}}{1-\alpha}$, i.e., $\boldsymbol{\omega}_{t}$ is bounded. Thus, $(1-3 \eta) \sum_{t=1}^{k}\left\|\boldsymbol{\omega}_{t+1}-\boldsymbol{\omega}_{t}\right\|_{\Theta}^{2} \leq s_{1}+$ $\eta^{k+1}\left(\left\|\boldsymbol{\omega}_{1}-\boldsymbol{\omega}^{*}\right\|_{\Theta}^{2}-\frac{\mu_{1}}{1-\alpha}\right)+\frac{\eta \mu_{1}}{1-\alpha}$. As $0<\eta<\frac{1}{3}$, we have

$$
\sum_{t=1}^{\infty}\left\|\boldsymbol{\omega}_{t+1}-\boldsymbol{\omega}_{t}\right\|_{\Theta}^{2}<\infty
$$

Thus, $\lim _{t \rightarrow \infty}\left(\boldsymbol{\omega}_{t+1}-\boldsymbol{\omega}_{t}\right)=0$. 
Then, we prove that $\left\|\boldsymbol{\omega}_{t}-\boldsymbol{\omega}^{*}\right\|_{\Theta}^{2}$ also converges by the similar analysis in [38, Theorem 6.3]. We also write it here for completeness.

Denote $\varphi_{t}=\max \left\{0,\left\|\boldsymbol{\omega}_{t}-\boldsymbol{\omega}^{*}\right\|_{\Theta}^{2}-\left\|\boldsymbol{\omega}_{t+1}-\boldsymbol{\omega}^{*}\right\|_{\Theta}^{2}\right\}$ and $\zeta_{t}=2 \eta\left\|\boldsymbol{\omega}_{t}-\boldsymbol{\omega}_{t-1}\right\|_{\Theta}^{2}$. We know that $\varphi_{t}$ is lower bounded. Recall (C.6), and we have $\varphi_{t+1} \leq \eta \varphi_{t}+\zeta_{t}$. Apply this relationship recursively, and we have

$$
\varphi_{t+1} \leq \eta^{t} \varphi_{1}+\sum_{i=0}^{t-1} \eta^{i} \zeta_{t-i}
$$

Adding both sides of (C.9) from $t=1 \rightarrow \infty$, we have

$$
\sum_{i=1}^{\infty} \varphi_{i} \leq \frac{\varphi_{1}}{1-\eta}+\frac{1}{1-\eta} \sum_{t=1}^{\infty} \zeta_{t}
$$

From (C.8), we know $\sum_{t=1}^{\infty} \zeta_{t}<\infty$. This implies that $\sum_{t=1}^{\infty} \varphi_{t}$ is bounded, non-decreasing and converges.

Consider another sequence $\left\{\left\|\omega_{t}-\omega^{*}\right\|_{\Theta}^{2}-\sum_{i=1}^{t} \varphi_{i}\right\}$, which is lower bounded. Moreover,

$$
\begin{aligned}
& \left\|\boldsymbol{\omega}_{t+1}-\boldsymbol{\omega}^{*}\right\|_{\Theta}^{2}-\sum_{i=1}^{k+1} \varphi_{i}=\left\|\boldsymbol{\omega}_{t+1}-\boldsymbol{\omega}^{*}\right\|_{\Theta}^{2}-\varphi_{k+1}-\sum_{i=1}^{t} \varphi_{i} \\
& \leq\left\|\boldsymbol{\omega}_{t+1}-\boldsymbol{\omega}^{*}\right\|_{\Theta}^{2}-\left\|\boldsymbol{\omega}_{t+1}-\boldsymbol{\omega}^{*}\right\|_{\Theta}^{2}+\left\|\boldsymbol{\omega}_{t}-\boldsymbol{\omega}^{*}\right\|_{\Theta}^{2}-\sum_{i=1}^{t} \varphi_{i} \\
& =\left\|\boldsymbol{\omega}_{t}-\boldsymbol{\omega}^{*}\right\|_{\Theta}^{2}-\sum_{i=1}^{t} \varphi_{i}
\end{aligned}
$$

This implies that $\left\{\left\|\omega_{t}-\omega^{*}\right\|_{\Theta}^{2}-\sum_{i=1}^{t} \varphi_{i}\right\}$ is a nonincreasing sequence and also converges. $\left\|\omega_{t}-\omega^{*}\right\|_{\Theta}^{2}$ is the sum of two convergent sequences, and also converges.

Since $\omega_{t}$ is bounded, it has a convergent subsequence $\omega_{n_{t}}$ converging to a point $\tilde{\boldsymbol{\omega}}^{*}$. From $\lim _{t \rightarrow \infty}\left(\boldsymbol{\omega}_{t+1}-\boldsymbol{\omega}_{t}\right)=0$, we have $\lim _{t \rightarrow \infty}\left(\boldsymbol{\omega}_{n_{t}+1}-\boldsymbol{\omega}_{n_{t}}\right)=0$ and $\lim _{t \rightarrow \infty}\left(\boldsymbol{\omega}_{n_{t}-1}-\boldsymbol{\omega}_{n_{t}}\right)=0$. Due to the continuity of the righthand side of (30), we have $\tilde{\omega}^{*}=$ $\left(\mathrm{Id}+\Theta^{-1} \mathcal{U}\right)^{-1} \tilde{\boldsymbol{\omega}}^{*}$. Thus, $\boldsymbol{\omega}^{*}$ is an equilibrium point of the sequence $\boldsymbol{\omega}_{t}$. This also implies that $\left\|\boldsymbol{\omega}_{t}-\tilde{\boldsymbol{\omega}}^{*}\right\|_{\Theta}^{2}$ converges. Because $\left\|\boldsymbol{\omega}_{n_{t}}-\tilde{\boldsymbol{\omega}}^{*}\right\|_{\Theta}^{2}$ converges to zero, $\left\|\boldsymbol{\omega}_{t}-\tilde{\boldsymbol{\omega}}^{*}\right\|_{\Theta}^{2}$ also converges to zero.

Based on Theorem 6, we have $\left(\boldsymbol{p}^{*}, \boldsymbol{b}^{*}\right)$ is the GNE of (7). This completes the proof.

\section{REFERENCES}

[1] Y. M. Chiang, "Building a better battery," Science, vol. 330, no. 6010, pp. 1485-1486, 2010.

[2] P. Mani, J. Lee, K. Kang, and Y. H. Joo, "Digital controller design via lmis for direct-driven surface mounted pmsg-based wind energy conversion system," IEEE Transactions on Cybernetics, pp. 1-12, 2019.

[3] Z. Wang, Y. Chen, S. Mei, S. Huang, and Y. Xu, "Optimal expansion planning of isolated microgrid with renewable energy resources and controllable loads," IET Renewable Power Generation, vol. 11, no. 7, pp. 931-940, 2017

[4] M. Tran, D. Banister, J. D. K. Bishop, and M. D. Mcculloch, "Realizing the electric-vehicle revolution," Nature Climate Change, vol. 2, no. 5, pp. 328-333, 2012.

[5] Y. Zhang, L. Chu, Y. Ou, C. Guo, Y. Liu, and X. Tang, "A cyberphysical system-based velocity-profile prediction method and case study of application in plug-in hybrid electric vehicle," IEEE Transactions on Cybernetics, pp. 1-12, 2019.

[6] D. M. Han and J. H. Lim, "Smart home energy management system using ieee 802.15.4 and zigbee," IEEE Transactions on Consumer Electronics, vol. 56, no. 3, pp. 1403-1410, 2010.
[7] S. Weng, D. Yue, C. Dou, J. Shi, and C. Huang, "Distributed eventtriggered cooperative control for frequency and voltage stability and power sharing in isolated inverter-based microgrid," IEEE transactions on cybernetics, no. 99, pp. 1-13, 2018.

[8] H. Zhang, D. Yue, C. Dou, K. Li, and X. Xie, "Event-triggered multiagent optimization for two-layered model of hybrid energy system with price bidding-based demand response," IEEE Transactions on Cybernetics, pp. 1-12, 2019.

[9] A. Dimeas, S. Drenkard, N. Hatziargyriou, S. Karnouskos, K. Kok, J. Ringelstein, and A. Weidlich, "Smart houses in the smart grid: Developing an interactive network," IEEE Electrification Magazine, vol. 2, no. 1, pp. 81-93, 2014.

[10] Y. Parag and B. K. Sovacool, "Electricity market design for the prosumer era," Nature Energy, vol. 1, no. 4, pp. 1-6, 2016.

[11] T. Morstyn, N. Farrell, S. J. Darby, and M. D. Mcculloch, "Using peer-to-peer energy-trading platforms to incentivize prosumers to form federated power plants," Nature Energy, vol. 3, no. 2, pp. 94-101, 2018.

[12] N. Liu, X. Yu, C. Wang, C. Li, L. Ma, and J. Lei, "Energy-sharing model with price-based demand response for microgrids of peer-to-peer prosumers," IEEE Trans. Power Syst., vol. 32, no. 5, pp. 3569-3583, 2017.

[13] Z. Wang, F. Liu, S. H. Low, C. Zhao, and S. Mei, "Distributed frequency control with operational constraints, part ii: Network power balance," IEEE Trans. Smart Grid, vol. 10, no. 1, pp. 53-64, Jan 2019.

[14] F. Dörfler, J. W. Simpson-Porco, and F. Bullo, "Breaking the hierarchy: Distributed control and economic optimality in microgrids," IEEE Trans. Control Network Syst., vol. 3, no. 3, pp. 241-253, 2016.

[15] Z. Wang, F. Liu, Y. Chen, S. H. Low, and S. Mei, "Unified distributed control of stand-alone dc microgrids," IEEE Trans. Smart Grid, vol. 10, no. 1, pp. 1013-1024, Jan 2019.

[16] Z. Wang, F. Liu, S. H. Low, P. Yang, and S. Mei, "Distributed load-side control: Coping with variation of renewable generations," Automatica, in press, 2019.

[17] N. Liu, X. Yu, C. Wang, and J. Wang, "Energy sharing management for microgrids with pv prosumers: A stackelberg game approach," IEEE Transactions on Industrial Informatics, vol. 13, no. 3, pp. 1088-1098, 2017.

[18] N. Liu, X. Yu, C. Wang, C. Li, L. Ma, and J. Lei, "Energy-sharing model with price-based demand response for microgrids of peer-to-peer prosumers," IEEE Transactions on Power Systems, vol. 32, no. 5, pp. 3569-3583, 2017.

[19] G. El Rahi, S. R. Etesami, W. Saad, N. B. Mandayam, and H. V. Poor, "Managing price uncertainty in prosumer-centric energy trading: A prospect-theoretic stackelberg game approach," IEEE Transactions on Smart Grid, vol. 10, no. 1, pp. 702-713, 2017.

[20] N. Liu, M. Cheng, X. Yu, J. Zhong, and J. Lei, "Energy-sharing provider for pv prosumer clusters: A hybrid approach using stochastic programming and stackelberg game," IEEE Transactions on Industrial Electronics, vol. 65, no. 8, pp. 6740-6750, 2018.

[21] A. Paudel, K. Chaudhari, C. Long, and H. B. Gooi, "Peer-to-peer energy trading in a prosumer-based community microgrid: A game-theoretic model," IEEE Transactions on Industrial Electronics, vol. 66, no. 8, pp. 6087-6097, 2018.

[22] C. Long, J. Wu, Y. Zhou, and N. Jenkins, "Peer-to-peer energy sharing through a two-stage aggregated battery control in a community microgrid," Applied energy, vol. 226, pp. 261-276, 2018.

[23] S. Cui, Y.-W. Wang, and J.-W. Xiao, "Peer-to-peer energy sharing among smart energy buildings by distributed transaction," IEEE Transactions on Smart Grid, vol. 10, no. 6, pp. 6491-6501, 2019.

[24] L. Chen, N. Liu, C. Li, and J. Wang, "Peer-to-peer energy sharing with social attributes: A stochastic leader-follower game approach," IEEE Transactions on Industrial Informatics, 2020.

[25] S. Cui, Y.-W. Wang, Y. Shi, and J.-W. Xiao, "A new and fair peer-to-peer energy sharing framework for energy buildings," IEEE Transactions on Smart Grid, 2020.

[26] H. Le Cadre, P. Jacquot, C. Wan, and C. Alasseur, "Peer-to-peer electricity market analysis: From variational to generalized nash equilibrium," European Journal of Operational Research, vol. 282, no. 2, pp. 753-771, 2020.

[27] P. Dutta and A. Boulanger, "Game theoretic approach to offering participation incentives for electric vehicle-to-vehicle charge sharing," in 2014 IEEE Transportation Electrification Conference and Expo (ITEC). IEEE, 2014, pp. 1-5.

[28] T. Tanaka, N. Li, and K. Uchida, "On the relationship between the vcg mechanism and market clearing," in 2018 Annual American Control Conference (ACC). IEEE, 2018, pp. 4597-4603. 
[29] Z. Fang, L. Huang, and A. Wierman, "Prices and subsidies in the sharing economy," Performance Evaluation, vol. 136, p. 102037, 2019.

[30] Y. Chen, S. Mei, F. Zhou, S. H. Low, W. Wei, and F. Liu, "An energy sharing game with generalized demand bidding: Model and properties," IEEE Transactions on Smart Grid, vol. 11, no. 3, pp. 2055-2066, 2020.

[31] Y. Chen, C. Zhao, S. H. Low, and S. Mei, "Approaching prosumer social optimum via energy sharing with proof of convergence," IEEE Transactions on Smart Grid, 2020.

[32] W. Qi, B. Shen, H. Zhang, and Z.-J. M. Shen, "Sharing demand-side energy resources-a conceptual design," Energy, vol. 135, pp. 455-465, 2017.

[33] P. Chakraborty, E. Baeyens, K. Poolla, P. P. Khargonekar, and P. Varaiya, "Sharing storage in a smart grid: A coalitional game approach," IEEE Transactions on Smart Grid, vol. 10, no. 4, pp. 4379-4390, 2018.

[34] J. Mei, C. Chen, J. Wang, and J. L. Kirtley, "Coalitional game theory based local power exchange algorithm for networked microgrids," Applied energy, vol. 239, pp. 133-141, 2019.

[35] L. Han, T. Morstyn, and M. McCulloch, "Incentivizing prosumer coalitions with energy management using cooperative game theory," IEEE Transactions on Power Systems, vol. 34, no. 1, pp. 303-313, 2019.

[36] S. Liang, P. Yi, and Y. Hong, "Distributed nash equilibrium seeking for aggregative games with coupled constraints," Automatica, vol. 85, pp. 179-185, 2017.

[37] X. Zeng, J. Chen, S. Liang, and Y. Hong, "Generalized nash equilibrium seeking strategy for distributed nonsmooth multi-cluster game," Automatica, vol. 103, pp. $20-26,2019$.

[38] P. Yi and L. Pavel, "An operator splitting approach for distributed generalized nash equilibria computation," Automatica, vol. 102, pp. 111121,2019

[39] P. Yi and L. Pavel, "Asynchronous distributed algorithms for seeking generalized nash equilibria under full and partial-decision information," IEEE transactions on cybernetics, in press, 2019.

[40] C. Yu, M. van der Schaar, and A. H. Sayed, "Distributed learning for stochastic generalized nash equilibrium problems," IEEE Transactions on Signal Processing, vol. 65, no. 15, pp. 3893-3908, Aug 2017.

[41] D. Paccagnan, B. Gentile, F. Parise, M. Kamgarpour, and J. Lygeros, "Nash and wardrop equilibria in aggregative games with coupling constraints," IEEE Transactions on Automatic Control, vol. 64, no. 4, pp. 1373-1388, April 2019.

[42] F. Salehisadaghiani and L. Pavel, "Distributed nash equilibrium seeking via the alternating direction method of multipliers," IFAC-PapersOnLine, vol. 50, no. 1, pp. 6166 - 6171, 2017.

[43] P. Yi and L. Pavel, "Distributed generalized nash equilibria computation of monotone games via double-layer preconditioned proximal-point algorithms," IEEE Transactions on Control of Network Systems, vol. 6, no. 1, pp. 299-311, 2018

[44] G. Belgioioso and S. Grammatico, "A distributed proximal-point algorithm for nash equilibrium seeking in generalized potential games with linearly coupled cost functions," in 2019 18th European Control Conference (ECC), June 2019, pp. 1-6.

[45] F. Salehisadaghiani, W. Shi, and L. Pavel, "Distributed nash equilibrium seeking under partial-decision information via the alternating direction method of multipliers," Automatica, vol. 103, pp. 27 - 35, 2019.

[46] L. Pavel, "Distributed gne seeking under partial-decision information over networks via a doubly-augmented operator splitting approach," IEEE Trans. Autom. Control, vol. 65, no. 4, pp. 1584-1597, 2020.

[47] D. Gadjov and L. Pavel, "A passivity-based approach to nash equilibrium seeking over networks," IEEE Transactions on Automatic Control, vol. 64, no. 3, pp. 1077-1092, 2018.

[48] H. Bauschke and P. L. Combettes, Convex Analysis and Monotone Operator Theory in Hilbert Spaces. Springer, 2017.

[49] S. Boyd and L. Vandenberghe, Convex optimization. Cambridge university press, 2004.

[50] F. Facchinei and C. Kanzow, "Generalized nash equilibrium problems," Annals of Operations Research, vol. 175, no. 1, pp. 177-211, 2010.

[51] R. Egging-Bratseth, T. Baltensperger, and A. Tomasgard, "Solving oligopolistic equilibrium problems with convex optimization," European Journal of Operational Research, vol. 284, no. 1, pp. 44-52, 2020.

[52] F. Alvarez and H. Attouch, "An inertial proximal method for maximal monotone operators via discretization of a nonlinear oscillator with damping," Set-Valued Analysis, vol. 9, no. 1-2, pp. 3-11, 2001.

[53] A. Wibisono, A. C. Wilson, and M. I. Jordan, "A variational perspective on accelerated methods in optimization," proceedings of the National Academy of Sciences, vol. 113, no. 47, pp. E7351-E7358, 2016.

[54] H. Attouch, Z. Chbani, J. Peypouquet, and P. Redont, "Fast convergence of inertial dynamics and algorithms with asymptotic vanishing viscosity," Mathematical Programming, vol. 168, no. 1-2, pp. 123-175, 2018.
[55] G. Qu and N. Li, "Accelerated distributed Nesterov gradient descent," IEEE Trans. Autom. Control, vol. 65, no. 6, pp. 2566-2581, 2020.

[56] W. H. Kersting, "Radial distribution test feeders," IEEE Transactions on Power Systems, vol. 6, no. 3, pp. 975-985, 1991.

[57] "Home of matpower," https://matpower.org/, August 2020.

[58] T. Erseghe, "Distributed optimal power flow using admm," IEEE transactions on power systems, vol. 29, no. 5, pp. 2370-2380, 2014

[59] Y. Han, L. Chen, Z. Wang, S. Mei, and W. Liu, "Fully distributed optimal power flow for unbalanced distribution networks based on admm," in 2016 IEEE International Conference on Power System Technology (POWERCON). IEEE, 2016, pp. 1-6.

[60] A. Makhdoumi and A. Ozdaglar, "Convergence rate of distributed admm over networks," IEEE Transactions on Automatic Control, vol. 62 no. 10 , pp. 5082-5095, 2017

[61] P. Yi, Y. Hong, and F. Liu, "Initialization-free distributed algorithms for optimal resource allocation with feasibility constraints and application to economic dispatch of power systems," Automatica, vol. 74, pp. 259-269, 2016.

[62] O. Mégel, T. Liu, D. J. Hill, and G. Andersson, "Distributed secondary frequency control algorithm considering storage efficiency," IEEE Transactions on Smart Grid, vol. 9, no. 6, pp. 6214-6228, 2017.

[63] H. Le Cadre, Y. Mou, and H. Höschle, "Parametrized inexact-admm to span the set of generalized nash equilibria: A normalized equilibrium approach," 2020.

[64] A. P. Ruszczyński and A. Ruszczynski, Nonlinear optimization. Princeton university press, 2006, vol. 13 .

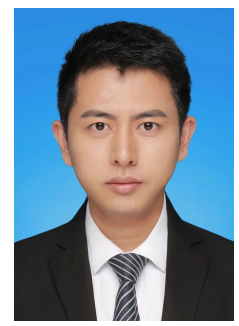

Zhaojian Wang (M'18) received the B.Sc. degree from Tianjin University in Electrical Engineering, Tianjin, China, in 2013, and the Ph.D. degree from Tsinghua University in Electrical Engineering, Beijing, China, in 2018. From 2016 to 2017, he was a visiting Ph.D. student at California Institute of Technology, CA, USA. From 2018 to 2020, he was a post-doctoral scholar at Tsinghua University. $\mathrm{He}$ is currently an assistant professor at Shanghai Jiao Tong University.

Dr. Zhaojian Wang's research interests include stability analysis, optimal control, microgrid planning and game theory based decision making in energy and power systems. He is an associate editor of IET Renewable Power Generation.

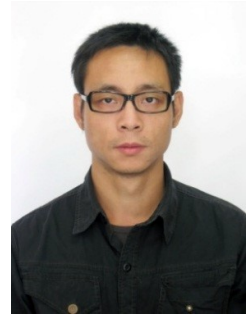

Feng Liu (M'12) received the B.Sc. and Ph.D. degrees in electrical engineering from Tsinghua University, Beijing, China, in 1999 and 2004, respectively. $\mathrm{He}$ is currently an Associate Professor of Tsinghua University. From 2015 to 2016, he was a visiting associate at California Institute of Technology, CA, USA.

Dr. Feng Liu's research interests include stability analysis, optimal control, robust dispatch and game theory based decision making in energy and power systems. He is the author/coauthor of more than 200 peer-reviewed technical papers and two books, and holds more than 20 issued/pending patents. He is an associated editor of several international journals including IEEE Transactions on Smart Grid and IEEE PES Letter. He also served as a guest editor of IEEE Transactions on Energy Conversion. 


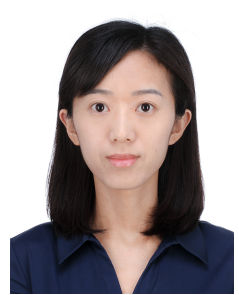

Zhiyuan Ma received her B.E. degree from the Shandong University of Science and Technology, Shandong, China, in 2011, the M.S. and Ph.D degrees in electrical engineering from Tsinghua University, Beijing, China, in 2014 and 2018, respectively. Currently, she works at the Global Energy Interconnection Development and Cooperation Organization (GEIDCO). Her research interests include climate change mitigation, energy and power systems.

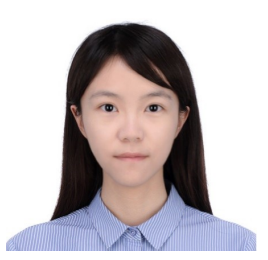

Yue Chen (S'15) received her B.E. and Ph.D. degrees in Electrical Engineering from Tsinghua University, Beijing, China, in 2015 and 2020, respectively; and her B.S. degree in Economics from Peking University, Beijing, China, in 2017. From 2018 to 2019, she was a visiting student at California Institute of Technology, Pasadena, CA, USA.

Her research interests include smart cities, sustainable energy, transactive energy, etc. based on algorithmic game theory, mathematical economics, and applied optimization.

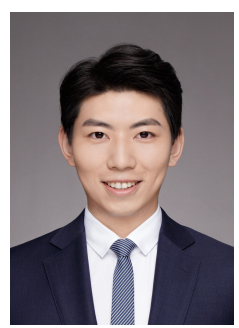

Mengshuo Jia (S'18) received his B.E. degree in Electrical Engineering from North China Electric Power University, BaoDing, China, in 2016. He is pursuing his $\mathrm{Ph} . \mathrm{D}$. degree in electrical engineering at Tsinghua University, Beijing, China. He is also a visiting student researcher with the Swiss Federal Institute of Technology, Zurich, Switzerland.

His research interests include probabilistic analytics and distributed computing in power systems.

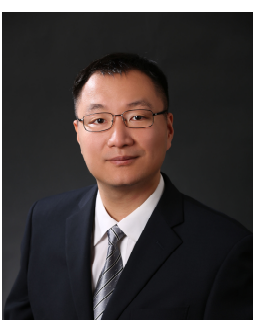

Wei Wei (SM'18) received the B.Sc. and Ph.D. degrees in electrical engineering from Tsinghua University, Beijing, China, in 2008 and 2013, respectively.

He was a Postdoctoral Research Associate with Tsinghua University from 2013 to 2015. He was a Visiting Scholar with Cornell University, Ithaca, NY USA, in 2014, and a Visiting Scholar with Harvard University, Cambridge, MA, USA, in 2015. He is currently an Associate Professor with Tsinghua University. His research interests include computational optimization and energy system economics. 